\title{
A GENERAL APPROACH TO THE OPTIMALITY OF MINIMUM DISTANCE ESTIMATORS
}

\author{
BY \\ P. W. MILLAR ${ }^{1}$
}

\begin{abstract}
Let $\theta$ be an open subset of a separable Hilbert space, and $\xi_{n}(\theta)$, $\theta \in \Theta$, a sequence of stochastic processes with values in a (different) Hilbert space $B$. This paper develops an asymptotic expansion and an asymptotic minimax result for "estimates" $\hat{\theta}_{n}$ defined by $\inf _{\theta}\left|\xi_{n}(\theta)\right|=\left|\xi_{n}\left(\hat{\theta}_{n}\right)\right|$, where $|\cdot|$ is the norm of $B$. The abstract results are applied to study optimality and asymptotic normality of procedures in a number of important practical problems, including simple regression, spectral function estimation, quantile function methods, min-chi-square methods, min-Hellinger methods, minimum distance methods based on $M$-functionals, and so forth. The results unify several studies in the literature, but most of the LAM results are new. From the point of view of applications, the entire paper is a sustained essay concerning the problem of fitting data with a reasonable, but relatively simple, model that everyone knows cannot be exact.
\end{abstract}

I. Introduction. This paper presents a rather general approach to the optimality properties of a broad class of estimators of the "minimum distance type". To see what this approach is, let us first consider several important, practical special cases.

Let us begin with the classic case. Suppose $X_{1}, \ldots, X_{n}$ are i.i.d. random variables, the distribution of $X_{i}$ being $P_{\theta}$, where $\left\{P_{\theta}, \theta \in \Theta\right\}$ is a family of probabilities on the line indexed by $\Theta$, an open subset of $R^{d}$. The statistical problem is to "estimate $\theta$ "; as pointed out several times in this paper, the phrase "estimate $\theta$ " as it has been used throughout much of the statistical literature is usually too vague to have a solid statistical meaning. However, for the purposes of these introductory heuristics let us suppose that we know what this means. Let $F_{\theta}$ be the cdf of $P_{\theta}$; let $\hat{F}_{n}$ be the empirical cdf. Ther $F_{\theta}, \hat{F}_{n}$ can be regarded as elements of several varieties of Banach spaces. For example, if $\mu$ is a finite measure on the line, $F_{\theta}, \hat{F}_{n}$ belong to $L^{2}(\mu)$ or to $L^{1}(\mu)$; if $F_{\theta}$ is continuous and a standard smoothing of $\hat{F}_{n}$ adopted, then $F_{\theta}, \hat{F}_{n}$ both belong to a Banach space of real bounded continuous functions with supremum norm. Therefore, let us denote by $B$ the Banach space in which $F_{\theta}, \hat{F}_{n}$ are alleged to lie, and by $|\cdot|_{B}$ the norm of $B$. The classic minimum distance estimate of $\theta$, based on $\hat{F}_{n}$, is then the point $\hat{\theta}_{n}$ that satisfies

$$
\inf _{\theta}\left|F_{\theta}-\hat{F}_{n}\right|_{B}=\left|F_{\theta}-F_{\hat{\theta}_{n}}\right|_{B}
$$

Received by the editors January 22, 1984.

1980 Mathematics Subject Classification. Primary 62F12; Secondary 62F35, 62G20.

Key wonds and phrases. Local asymptotic minimax, minimum distance estimator, robust estimator, Cramer-von Mises statistic, minimum chi-square, differentiable statistical functional, abstract Wiener space.

${ }^{1}$ Research supported by National Science Foundation Grant MCS80-02648. 
Here, and elsewhere in this introduction, we ignore the problems of existence and uniqueness of such a $\hat{\theta}_{n}$ : these difficulties will be treated in subsequent sections.

There are innumerable variants of the recipe (1.1). For example, one could let $\phi_{\theta}$ be the characteristic function (ch.f.) of $P_{\theta}$, and $\hat{\phi}_{n}$ the ch.f. of $\hat{F}_{n}$, and define another minimum distance estimate $\hat{\theta}_{2 n}$ by

$$
\inf _{\theta}\left|\phi_{\theta}-\hat{\phi}_{n}\right|_{B}=\left|\phi_{\hat{\theta}_{2, n}}-\hat{\phi}_{n}\right|_{B}
$$

where, as before, there is quite a bit of freedom in the choice of $B$. As another possibility, one could let $F_{\theta}^{-1}$ denote the quantile function of $P_{\theta}$ : the function on $[0,1]$ defined by $F_{\theta}^{-1}(t)=\inf \{s: F(s) \geq t\}$, and then one could let $\hat{F}_{n}^{-1}$ be the quantile function of $\hat{F}_{n}$. Yet another minimum distance estimate, $\hat{\theta}_{3, n}$, of $\theta$ is then possible via the recipe

$$
\inf _{\theta}\left|\hat{F}_{n}^{-1}-F_{\theta}^{-1}\right|_{B}=\left|\hat{F}_{n}^{-1}-F_{\hat{\theta}_{3, n}}^{-1}\right|_{B}
$$

Again there is much freedom in the choice of $B$. All of these estimators have been considered in the literature; for some new ones in a similar vein, which have additional interesting properties, see $\S 13$. More complexity is possible-and even useful: one can replace the norm ||$_{B}$ by a family of norms ||$_{\theta}$; this device leads to the well-known weighted Cramer-von Mises estimates.

The illustrations of the preceding paragraph dealt with estimation in a certain i.i.d. framework. There are other situations, of great practical interest, which share exactly the same abstract structure. For a first example, let $X_{1}, \ldots, X_{n}$ be a stationary Gaussian sequence with mean 0 , spectral measure $P_{\theta}$, where $\theta \in \Theta$, an open subset of $R^{d}$. Again one wants to "estimate $\theta$ ". Let

$$
\hat{F}_{n}(t)=(2 \pi n)^{-1} \int_{0}^{+}\left|\sum_{1}^{n} X_{0} e^{-i y k}\right|^{2} d y .
$$

Let $F_{\theta}(t)$ be the cumulative of the spectral measure $P_{\theta}$ and bring in an appropriate Banach space $B$ as in the preceding example. Then a minimum distance estimate $\hat{\theta}_{n}$ of $\theta$ is defined by

$$
\inf _{\theta}\left|F_{\theta}-\hat{F}_{n}\right|_{B}=\left|F_{\hat{\theta}_{n}}-\hat{F}_{n}\right|_{B}
$$

As one further example, consider a "simple regression" model. Let $X_{n 1}, \ldots, X_{n n}$ be independent random variables. Assume a distribution $F$ is given and there are known numbers $c_{n i}, 1 \leq i \leq n$, such that $\left\{X_{n i}-\theta c_{n i}, 1 \leq i \leq n\right\}$ is an i.i.d. $(F)$ sequence for some $\theta$. Again the problem is to estimate $\theta$. Define a random function

$$
\xi_{n}(\theta ; t)=\sum d_{n i}\left[I\left\{X_{n i} \leq t+\theta c_{n i}\right\}-F(t)\right]
$$

where $\left\{d_{n i}, 1 \leq i \leq n\right\}$ is a sequence of real numbers. Bring in, yet again, some Banach space $B$, so that $\xi_{n}(\theta ; \cdot) \in B$ for each $\theta$. A minimum distance estimate of $\theta, \hat{\theta}_{n}$, is then defined by

$$
\inf _{n}\left|\xi_{n}(\theta ; \cdot)\right|_{B}=\left|\xi_{n}\left(\hat{\theta}_{n} ; \cdot\right)\right|_{B}
$$

The estimator (1.5) has been partially analyzed in the literature. The estimate (1.4) appears to be new (see $\S \S 10,11$ ). 
One of the main points of this paper is that, under mild, natural conditions, all of the estimates described above-and many more-share a common, very simple abstract structure asymptotically. This common structure enables one to deduce immediately the asymptotic normality and, just as important, a strong asymptotic optimality.

The abstract structure is of the following sort. One is given a sequence $\xi_{n}(\theta)$ of $B$-valued stochastic processes ('time' parameter is $\theta \in \Theta$ ). The statistical problem is to compute $\hat{\theta}_{n}$ satisfying

$$
\inf _{\theta}\left|\xi_{n}(\theta)\right|_{B}=\left|\xi_{n}(\hat{\theta})\right|_{B}
$$

In (1.1), $\xi_{n}(\theta)$ could be $F_{\theta}-\hat{F}_{n}$; in (1.2), $\xi_{n}(\theta)$ could be $\hat{\phi}_{n}-\phi_{\theta}\left(\right.$ or $\sqrt{n}\left(\hat{\phi}_{n}-\phi_{\theta}\right)$ ), and so forth. Under reasonable smoothness conditions on the sample functions $\theta \rightarrow$ $\xi_{n}(\theta)$, it is possible to give a very simple characterization of $\xi\left(\hat{\theta}_{n}\right)$, a characterization that leads swiftly in all of the applications to an asymptotic normality result for $\hat{\theta}_{n}$. These matters are discussed in $\S \S 2,3$. There is a long history of minimum distance estimation; see Parr for a survey. The development here draws on the analyses of Bolthausen, Millar, Pollard and Wolfowitz.

The optimality properties of such minimum distance estimators are harder to describe. To get a feel for it, return to the classic case (1.1). Let us suppose $X_{1}, \ldots, X_{n}$ are i.i.d. $G$, but $G$ is presumed to be possibly different from any of the $P_{\theta}$ 's. This fact notwithstanding, the basic statistical goal is defined to being that of fitting the "model" $\left\{P_{\theta}\right\}$ as "best as one can" to the data. There are several different situations in which one might desire to do this. One possibility is to assume that the $\left\{P_{\theta}\right\}$ model would have been fairly accurate, except somewhere along the line the data was subjected to "contamination" (thus altering the distributions slightly); such contamination could be due to roundoff errors, clerical errors, and so forth. Alternatively, one might be certain that model is inaccurate, but not too bad, and desire to use it because (say) it has an appealing simplicity that aids in getting a feel for the basic shape of the data. The precise notion of 'best fit' to be employed should be determined by your statistical goals. If you want to estimate a few probabilities, then (1.1) might be appropriate; if you wanted to estimate a few quantiles, perhaps (1.3) would be better. There are, evidently, a large number of choices, and a fair portion of this paper is devoted to explaining some of the consequences of a particular choice. The optimality results of this paper are elaborated in a framework where it is agreed that the statistical goal is one of "best fit"; within such a framework, estimators of the type suggested by $\hat{\theta}_{1 n}, \hat{\theta}_{2 n}, \hat{\theta}_{3 n}$, and many others, are shown to have a desirable stability property, called local asymptotic minimaxity (LAM). Roughly speaking, the optimality property asserts that estimators do not deteriorate when the actual data distribution departs somewhat from those given by the model: i.e., the minimum distance estimators are robust.

For the classic case one can formulate the estimation problem to be solved as follows: similar formulations extend to all other applications. The observations $X_{1}, \ldots, X_{n}$ are assumed i.i.d., with unknown distribution $G$. Define a functional $G \rightarrow \theta(G)$ by

$$
\inf _{\theta}\left|G-F_{\theta}\right|_{B}=\left|G-F_{\theta(G)}\right|_{B}
$$


Then the goal is to estimate $\theta(G)$, where $G$ is the actual data distribution. Thus, we estimate the $\theta \in \Theta$ that ensures the "best fit" of the model to the data, where (in the present case) 'best fit' is defined by (1.6). We emphasize again, that there are many choices for the notion of 'best fit', and the one you actually decide on depends upon what use you are trying to make of the data. However, given that your goal is that of best fit, it is quite irrelevant to evaluate your estimates on the basis of the criterion of 'least asymptotic variance': estimators with small asymptotic variance will not necessarily ensure your announced goal of 'best fit'. This should be kept in mind while perusing the optimality results below.

The paper is organized as follows. $§ \S 2,3$ give the basic asymptotic expansion that ensures (in all the examples treated) asymptotic normality of the minimum distance estimators. $\S \S 4,5$ give, in abstract form, the basic optimality result. $\S \S 6-$ 14 discuss a wide variety of applications. You may complain initially about the relatively high level of abstraction in the basic development-especially $\S \S 4,5$. On the other hand, once you read through the large and varied selection of useful applications, I think you will find it not unreasonable. $\S 15$ gives a couple of proofs omitted from $\S \S 2,3$.

The development of this paper unifies several results in the literature and provides a fair number of new results. The LAM results in the sections concerning spectral functions, quantile functions, min-Hellinger methods, the variety of methods in $\S 13$, the simple regression problem, possibly also $\S 8$, appear here for the first time. In most of these applications (except $\S \S 11,13$ ), asymptotic normality was either known or part of the folklore.

II. A basic asymptotic expansion. This section contains the basic asymptotic representation of minimum distance estimators which will be specialized in the applications of subsequent sections. That this level of abstraction is necessary can be seen quickly on reviewing at the same time (e.g.) the spectral function example and the regression example in $\S 1$.

To describe the result, let $\theta$ be a separable Hilbert space and fix $\theta_{0} \in \theta$. Let $\left(\Omega_{n}, \mathcal{F}_{n}, P_{n}\right)$ be a sequence of probability spaces, and let $\left\{\xi_{n}(\theta, \omega), \theta \in \Theta\right\}$ be a sequence of stochastic processes on $\left(\Omega_{n}, \mathcal{F}_{n}\right)$; here $\omega \in \Omega_{n}$. The processes $\xi_{n}(\theta, \omega)$ are assumed to have their values in a separable Hilbert space $B$ with norm ||$_{B}$. It is important that, so far, no hypotheses are placed on the probabilities $P_{n}$; in some useful applications (which show the Fréchet differentiability of certain functionals), $P_{n}$ is, for each $n$, concentrated at a single point.

Fix $\theta_{0} \in \boldsymbol{\Theta}$. Introduce the following hypotheses and definitions.

(2.1) Hypothesis of identifiability. As $c \uparrow \infty$, then, under $P_{n}$,

$$
\inf _{n} \inf _{\left|\theta-\theta_{0}\right|>c}\left|\xi_{n}(\theta ;)\right|_{B} \rightarrow \infty
$$

Measurability problems here can be avoided by taking a separable version of the process $\xi_{n}(\theta, \cdot)$ (cf. Doob (1953)). Many refinements of this hypothesis are possiblewith substantial increase in the level of tedium; for example, the inf need only be taken over "sufficiently large" $n$, an easy reformulation left to the reader. 
The second hypothesis is

(2.2) Differentiability. There exists a continuous linear map $T: \Theta \rightarrow B$ and a sequence of real numbers $\delta_{n}$ such that, for all $c$,

$$
\sup _{\left|\theta-\theta_{0}\right|<c}\left|\xi_{n}(\theta)-\xi_{n}\left(\theta_{0}\right)-\delta_{n} T\left(\theta-\theta_{0}\right)\right|_{B}=o_{P_{n}}(1)
$$

under $P_{n}$. Here $\xi_{n}$ is random (i.e., depends on $\omega \in \Omega$ ) but $T$ is assumed nonrandom.

When $\Theta=R^{d}$, one can endow $\Theta$ with the usual basis $\left\{\varepsilon_{1}, \ldots, \varepsilon_{d}\right\}$, so $\theta=\sum \theta_{i} \varepsilon_{i}$. Set $\eta_{i}=T\left(\varepsilon_{i}\right), \eta=\left(\eta_{1}, \eta_{2}, \ldots, \eta_{d}\right)$. Then

$$
T(\theta)=\langle\theta, \eta\rangle=\sum \theta_{i} \eta_{i}
$$

(2.3) Definition. The derivative $T$ is nonsingular if, for all $\theta,|T(\theta)| \geq b|\theta|$ for some $b>0$.

In the case that $\theta=R^{d}$, this assumption is equivalent to the condition that the $\eta_{i}$ in (2.2a) be linearly independent. The third assumption is

(2.4) Boundedness. Under $\left\{P_{n}\right\}$ the $B$-valued random variables $\xi_{n}\left(\theta_{0}\right)$ are norm bounded in probability: for each $\varepsilon>0$ there exists $c$ such that

$$
P_{n}\left\{\left|\xi_{n}\left(\theta_{0}\right)\right|_{B}>c\right\} \leq \varepsilon
$$

for all $n$. Alternatively, one could simply say that the $P_{n}$-distributions of the real random variables $\left|\xi_{n}\left(\theta_{0}\right)\right|_{B}$ are tight.

In many examples a stronger hypothesis holds.

(2.5) Convergence. Under $P_{n}$, the $B$-valued random elements $\xi_{n}\left(\theta_{0}\right)$ converge weakly on $B$ to a random element $W$.

This hypothesis, of course, implies (2.4); but in several applications it is not satisfied.

(2.6) Definition. Define $B_{\eta}$ to be the subspace of $B$ spanned by $\{T(\theta), \theta \in \Theta\}$ and define $\pi$ to be orthogonal projection of $B$ to $B_{\eta}$.

Define the minimum norm estimate $\hat{\theta}_{n}$ to be any point $\hat{\theta} \in \Theta$ satisfying

$$
\inf \left|\xi_{n}(\theta)\right|_{B}=\left|\xi_{n}\left(\hat{\theta}_{n}\right)\right|_{B}
$$

There are, at this point, problems of existence of $\hat{\theta}_{n}$; under assumptions (2.1), (2.2), (2.4) these problems disappear asymptotically. The worried reader can instead consider any $\hat{\theta}_{n}$ that achieves the inf in (2.6), with an error of $n^{-2}: \hat{\theta}_{n}$ should satisfy $\inf _{\theta}\left|\xi_{n}(\theta)\right|_{B} \geq\left|\xi_{n}\left(\hat{\theta}_{n}\right)\right|_{B}-n^{-2}$. Now there are no existence problems; however, since asymptotically we do have existence and uniqueness, we shall, for notational simplicity, proceed as though the inf were achieved.

The basic asymptotic expansion can now be stated.

(2.8) THEOREM. Assume the hypotheses of identifiability, differentiability, and boundedness. Define $\hat{\theta}_{n}$ by (2.7) and $\pi$ by (2.6). Then

$$
\xi_{n}\left(\hat{\theta}_{n}\right)=(1-\pi) \xi_{n}\left(\theta_{0}\right)+o_{P_{n}}(1) .
$$

As one might expect, a result this general has a crude proof based only on elementary considerations. This fact does not vitiate its utility. To see heuristically that the form of the result is reasonable, note that $(2.1),(2.4)$ suggest that $\hat{\theta}_{n}$, which 
minimizes the norm, must be in a vicinity of $\theta_{0}$. This being so, the differentiability assumption (2.2) implies

$$
\begin{aligned}
\left|\xi_{n}(\theta)\right|^{2} & =\left|\xi_{n}(\theta)-\xi_{n}\left(\theta_{0}\right)+\xi_{n}\left(\theta_{0}\right)\right|^{2} \doteq\left|\xi_{n}\left(\theta_{0}\right)+\delta_{n} T\left(\theta-\theta_{0}\right)\right|^{2} \\
& =\left|\pi \xi_{n}\left(\theta_{0}\right)+\delta_{n} T\left(\theta-\theta_{0}\right)\right|^{2}+\left|(1-\pi) \xi_{n}\left(\theta_{0}\right)\right|^{2}
\end{aligned}
$$

so that the minimum in $\theta$ should be at $\hat{\theta}_{n}$ satisfying $\pi \xi_{n}\left(\theta_{0}\right)+\delta_{n} T\left(\hat{\theta}_{n}-\theta_{0}\right)=0$. This means that

$$
\begin{aligned}
\xi\left(\hat{\theta}_{n}\right) & =\xi_{n}\left(\theta_{0}\right)+\xi_{n}\left(\hat{\theta}_{n}\right)-\xi_{n}\left(\theta_{0}\right) \\
& \doteq \xi_{n}\left(\theta_{0}\right)+\delta_{n} T\left(\hat{\theta}_{n}-\theta_{0}\right)=\xi_{n}\left(\theta_{0}\right)-\pi \xi_{n}\left(\theta_{0}\right),
\end{aligned}
$$

from which the result follows.

This result may be translated into a result involving $\hat{\theta}_{n}$ itself. To develop such a result, assume $T$ is nonsingular.

(2.10) COROLlary. Assume, in addition to the hypotheses of (2.8), that $T$ is nonsingular. Then

$$
\delta_{n}\left(\hat{\theta}_{n}-\theta_{0}\right)=-T^{-1} \circ \pi \xi_{n}\left(\theta_{0}\right)+o(1) .
$$

The corollary is immediate from (2.8).

The minimum point $\hat{\theta}_{n}$ is asymptotically unique in the sense that if $\tilde{\theta}_{n}$ is any other such point (at time $n$ ) then $\left|\hat{\theta}_{n}-\tilde{\theta}_{n}\right|=o(1)$.

The proof of (2.8) appears in $§ 15$. To facilitate subsequent applications, let us record the following refinements.

(2.12) Evaluation of $T^{-1} \circ \pi$. Assume $T$ is nonsingular. If $x \in B, x$ is the unique element of $B$ of the form $\pi x=T \theta_{x}\left(\theta_{x} \in \Theta\right)$ satisfying

$$
\inf _{\theta}|x-T \theta|_{B}^{2}=|x-\pi x|_{B}^{2}=\left|x-T \theta_{x}\right|_{B}^{2} .
$$

Using the fact that at the minimum point the functional $\theta \rightarrow|x-T \theta|^{2}$ must have derivative 0 , together with the form of the derivative at $\theta$ as $2\left\langle T^{*} x-T^{*} T \theta, \cdot\right\rangle$, one finds

$$
\theta_{x}=K^{-1} T^{*} x, \quad K=T^{*} T, \quad \pi x=T K^{-1} T^{*} x,
$$

where $T^{*}$ is the adjoint of $T$. The operator $K^{-1}$ exists and is continuous, because of the nonsingularity hypothesis.

If $\xi_{n}\left(\theta_{0}\right)$ converges in $B$ to a Gaussian random element $W$ with mean 0 and covariance operator $S$, then evidently, by $(2.11)$,

$$
\delta_{n}\left(\hat{\theta}_{n}-\theta_{0}\right) \Rightarrow K^{-1} T^{*} W
$$

a Gaussian random element with mean 0 and covariance operator

$$
\Gamma S \Gamma^{*}, \quad \Gamma=K^{-1} T^{*} \text {. }
$$

In all applications but one, $\theta$ is finite dimensional. To get a more explicit description in this case of $(2.14),(2.15)$, adopt the representation of $T$ given by $(2.2 \mathrm{a})$ :

$$
T \theta=\sum \theta_{i} \eta_{i} .
$$


Also, in all applications, $B$ is an $L^{2}(\mu)$ space of $R^{d}$. Assuming this framework, suppose $\xi_{n}\left(\theta_{n}\right)$ converges in $L^{2}$ to a Gaussian process $W$, where $E W(s)=0$, $E W(s) W(t)=R(s, t), s, t$ in $R^{d}$. Define a matrix $A=\left(A_{i j}\right)$ by

$$
A_{i j}=E\left\langle\eta_{i}, W\right\rangle_{B}\left\langle\eta_{j}, W\right\rangle_{B}=\iint \eta_{i}(s) R(s, t) \eta_{j}(t) \mu(d s) \mu(d t),
$$

and a matrix $C=\left(C_{i j}\right)$ by

$$
C_{i j}=\left\langle\eta_{i}, \eta_{j}\right\rangle_{B}
$$

Then $C^{-1}$ exists by nonsingularity. Specialization of (2.14), (2.15) to the present finite-dimensional case leads easily to

$$
\delta_{n}\left(\hat{\theta}_{n}-\theta_{0}\right) \Rightarrow Z
$$

where

$$
Z \text { is } N\left(0, C^{-1} A C\right) \text {, }
$$

III. Expansion for processes scaled by $n^{1 / 2}$. A number of the examples of this paper involve a particular case of the result of $\S 2$ : processes scaled by $n^{1 / 2}$. For ease of subsequent applications, we single out this case here.

Here $\Theta$ is an open subset of a Hilbert space, $\left\{P_{n}\right\}$ is a sequence of probabilities on $\left(\Omega_{n}, \mathcal{F}_{n}\right)$, and $B$ is a separable Hilbert space. For each $\theta$, let $\xi_{n}(\theta, \omega)$ be a $B$-valued random variable. Define $\hat{\theta}_{n}$ by

$$
\inf _{\theta \in \Theta}\left|\xi_{n}(\theta)\right|=\left|\xi_{n}\left(\hat{\theta}_{n}\right)\right|
$$

Fix $\theta_{0} \in \boldsymbol{\theta}$ and introduce the following variants of the hypotheses of $\S 2$.

(3.2) Identifiability. For every $\varepsilon, c>0$ there exists $\delta>0$ such that

$$
P_{n}\left\{\omega: \inf _{\theta:\left|\theta-\theta_{0}\right|>c}\left|\xi_{n}(\theta, \omega)-\xi_{n}\left(\theta_{0}, \omega\right)\right|>\delta\right\} \geq 1-\varepsilon
$$

for all $n$.

In several important applications, $\xi_{n}(\theta)-\xi_{n}\left(\theta_{0}\right)$ is free of $\omega$ and $n$, so the formulation is then much simpler.

(3.3) Boundedness. Under the probabilities $P_{n}$ the $B$-valued random variables $n^{1 / 2} \xi_{n}\left(\theta_{0}\right)$ are norm bounded in probability. As in $\S 2$, there is a stronger hypothesis, satisfied in most examples:

(3.4) Convergence. The $B$-valued random variables $n^{1 / 2} \xi_{n}\left(\theta_{0}\right)$ converge in distribution to a $B$-valued random variable $W$.

The third hypothesis is

(3.5) Differentiability. There exists a continuous linear operator $T=T_{\theta_{0}}$ from span $\Theta$ to $B$ such that

$$
\xi_{n}(\theta, \omega)=\xi_{n}\left(\theta_{0}, \omega\right)+T\left(\theta-\theta_{0}\right)+o_{P}\left(\left|\theta-\theta_{0}\right|\right) .
$$

The definition that $T$ be nonsingular is the same as in $\S 2$.

As in $\S 2$ let $B_{\eta}$ be the subspace of $B$ spanned by $T(\theta)$ and let $\pi$ be the orthogonal projection from $B$ to $B_{\eta}$. 
(3.6) THEOREM. Assume identifiability, boundedness, and differentiability $((3.2),(3.3),(3.5))$ with nonsingular derivative. Define $\hat{\theta}_{n}$ to be the minimum norm estimate (3.1). Then with probability approaching 1 as $n \rightarrow \infty, \hat{\theta}_{n}$ exists and is unique. Moreover,

$$
\begin{aligned}
\xi_{n}\left(\hat{\theta}_{n}\right) & =(1-\pi) \xi_{n}\left(\theta_{0}\right)+o\left(n^{-1 / 2}\right), \\
\hat{\theta}_{n}-\theta_{0} & =-T^{-1} \circ \pi \circ \xi_{n}\left(\theta_{0}\right)+o\left(n^{-1 / 2}\right) .
\end{aligned}
$$

If, in addition, the hypothesis of convergence holds,

$$
\begin{aligned}
& n^{1 / 2}\left[\xi_{n}\left(\hat{\theta}_{n}\right)-\xi_{n}\left(\theta_{0}\right)\right] \Rightarrow \pi \circ W \quad \text { in } B, \\
& n^{1 / 2}\left[\hat{\theta}_{n}-\theta_{0}\right] \Rightarrow-T^{-1} \circ \pi \circ W \quad \text { in } R^{d} .
\end{aligned}
$$

As in $\S 2$, if $W$ is Gaussian, so are the limits just mentioned and the evaluation of $T^{-1} \circ \pi$ can be found in (2.12).

Since we are interested in this paper mainly in the structural aspects of the problems treated, we use the more explicit evaluation (2.12) only in a few cases.

To prove the result just stated, let $\xi_{n}(\theta)$ be given and define

$$
\xi_{n}^{0}(\theta)=n^{1 / 2}\left[\xi_{n}\left(\theta_{0}+\theta n^{-1 / 2}\right)\right] .
$$

With modest effort, one sees that the hypotheses of $(2.8),(2.11)$ hold for $\xi_{n}^{0}$; then application of the result of (2.8) yields (3.6).

IV. The basic asymptotic minimax theorem. This section recalls the basic Hajek-Le Cam asymptotic minimax theorem, with the purpose of applying it efficiently to the optimality considerations of subsequent sections. This result is given in (4.9), after preliminaries on the theory of convergent experiments and on abstract Wiener spaces.

Let $\Theta$ be an arbitrary index set, and let $\left\{P_{\theta}, \theta \in \Theta\right\}$ be a family of probabilities on a measure space $(S, S)$. The triple $E=\left\{P_{\theta},(S, S), \theta \in \Theta\right\}=\left\{P_{\theta}\right\}$ is a statistical experiment.

Suppose, temporarily, that $\Theta$ is finite, $\Theta=\left\{\theta_{1}, \ldots, \theta_{d}\right\}$. The canonical measure $\mu_{E}$ of such an experiment $E$ is the measure on the unit simplex of $R^{d}$ given by the following recipe: if $M=\sum P_{\theta}$ and $m=\left(m_{1}, \ldots, m_{d}\right)$, where $m_{i}=d P_{\theta_{i}} / d M$, then $\mu_{E}$ is the distribution of the vector $m$. Suppose $E^{n}=\left\{P_{\theta}^{n}, \theta \in \Theta\right\}$ is a sequence of experiments indexed by $\Theta$, assumed finite. Then $E^{n}$ converges to $E$ if $\mu_{E^{n}}$, the canonical measure of $E^{n}$, converges weakly in $R^{d}$ to $\mu_{E}$. Statistical significance of canonical measures was pointed out by Blackwell (1953); the notion of convergence of experiments just defined is due to Le Cam (1964).

Next, suppose $\Theta$ is completely arbitrary. Let $E^{n}, E$ be statistical experiments indexed by $\Theta$. $E^{n}$ converges to $E$ if, for every finite subset $\Theta_{0} \subset \Theta$, the statistical experiments $\left\{P_{\theta}^{n}, \theta \in \Theta_{0}\right\}$ converge to $\left\{P_{\theta}, \theta \in \Theta_{0}\right\}$ in the sense just defined for finite $\Theta_{0}$. Le Cam (1964) has given several beautiful equivalences for this mode of convergence.

It is extremely painful to check convergence of canonical measures in general. In this respect, the following lemma is of some help; indeed, all of the convergence results used below can be checked by means of this lemma. 
(4.1) LEMMA. Let $E^{n}, E$ be statistical experiments indexed by $\Theta$. Suppose there exists $\theta_{0} \in \Theta$ such that each $P_{\theta}$ is absolutely continuous with respect to $P_{\theta_{0}}$ and $P_{\theta}^{n}$ is contiguous to $P_{\theta_{0}}^{n}$. For a finite subset $\Theta_{0}$ of $\Theta$, let $\mu_{00}$ denote the distribution of the vector $\left\{d P_{\theta} / d P_{\theta_{0}}: \theta \in \Theta_{0}\right\}$ under $P_{\theta_{0}}$, and define $\mu_{00}^{n}$ similarly for $E^{n}$. If, for each $\Theta_{0}, \mu_{00}^{n}$ converges weakly to $\mu_{00}$, then $E^{n}$ converges to $E$.

(4.2) REMARK. Many applications proceed by showing that the distribution of $\log d P_{\theta}^{n} / d P_{\theta_{0}}^{n}$ converges to that of $\log d P_{\theta} / d P_{\theta_{0}}$. Thus, in many statistical applications, the convergence theory of Le Cam is equivalent to certain simple convergences of the likelihood ratios.

The following example appears in some of the applications and is an important example of convergence experiments; it can be checked by routine use of (4.1).

(4.3) EXAMPLE. Let $F$ be a fixed distribution on the line with density $f$ with respect to Lebesgue measures. Let $h_{0}$ be a fixed real function and define, for real $\theta$

$$
f\left(\theta h_{0} ; x\right)=f(x)\left[1+\theta h_{0}(x)\right] .
$$

Assume (a) $\int f(x) h_{0}(x) d x=0$, and (b) $f\left(\theta h_{0} ; x\right) \geq 0$ for all small $\theta$. Then, for all small $\theta, f\left(\theta h_{0} ; \cdot\right)$ is a probability density. Let $P_{\theta}^{n}$ be the $n$-fold product measure of $f\left(\theta n^{-1 / 2} h_{0} ; \cdot\right)$; this will be defined for all large $n$. Then $\left\{P_{\theta}^{n}, \theta \in R^{\prime}\right\}$ converges to $\left\{P_{\theta}, \theta \in R^{1}\right\}$, where $P_{\theta}$ is the measure on the line with mean $\theta\left|h_{0}\right|$ and variance 1 , where $\left|h_{0}\right|^{2}=\int h_{0}^{2}(x) f(x) d x$.

For our applications, a modest extension of Example (4.3) is required. To describe it let $H$ be a separable Hilbert space, $B$ a separable Banach space, and $\tau$ a mapping of $H$ to $B$ that is continuous,linear, and one-to-one. Let $Q_{0}$ be the cylinder measure on $B$ with characteristic functional

$$
\phi(m)=\exp \left\{-\left|\tau^{*} m\right|_{H}^{2}\right\},
$$

where $m \in B^{*}$, the dual of $B, \tau^{*}$ is the adjoint of $\tau$, and $\left.\right|_{H}$ is the norm of $H$. Assume that $Q_{0}$ is countably additive on the Borel sets of $B$. That is, the triple $(\tau, H, B)$ is an abstract Wiener space. Slightly more detail giving the statistical relevance of such a construct may be found in Millar (1979). Here are two examples.

(4.5) EXAMPLE. $H=R^{k}, \tau h=\Gamma h$, where $\Gamma$ is a nonsingular matrix, and $B=R^{k}$. Then $Q_{0}$ is the normal distribution on $R^{k}$ with mean 0 and covariance $\Gamma \Gamma^{t}$.

(4.6) ExAMPLE. Let $F$ be a probability on the line with density $f$ with respect to $m$. Define

$$
H=\left\{h: \int h^{2} f d m<\infty, \int h f=0, \text { support } h \subset \text { support } f\right\} ;
$$

then $H$ is a Hilbert space with inner product $\left\langle h_{1}, h_{2}\right\rangle=\int h_{1} h_{2} f d m$. Define $\tau$, a mapping of $h$ to real functions on the line, by

$$
(\tau h)(t)=\int^{t} h(s) f(s) m(d s) .
$$

Let $\mu$ be a finite measure on the line; then $\tau h \in L^{2}(\mu)$. Define $B$ to be the closure in $L^{2}(\mu)$ of $\tau H$. Let $Q_{0}$ be the distribution of the stochastic process $\left\{W^{0}(F(t))\right\}$, where $\left\{W^{0}(s), 0<s<1\right\}$ is the usual Brownian bridge on $[0,1]$. Then $(\tau, H, B)$ 
is an abstract Wiener space since $Q_{0}$ is countably additive on $B$. This is the key example for most of the applications.

(4.7) Definition. Let $(\tau, H, B)$ be an abstract Wiener space and $Q_{0}$ the measure on $B$ with ch.f (4.4). Define measures $Q_{h}, h \in H$, on $B$ by $Q_{h}(A)=$ $Q_{0}(A-\tau h)$. The statistical experiment $\left\{Q_{h}, h \in H\right\}$ is called the standard Gaussian shift family for $(\tau, H, B)$.

For checking convergence of experiments to some standard Gaussian shift experiment $\left\{Q_{h}\right\}$ by the method of Lemma (4.1), it is necessary to know that, if $h_{1}, \ldots, h_{d} \in H$, then the vector $\left\{\log d Q_{h_{i}} / d Q_{0}: 1 \leq i \leq d\right\}$ has, under $Q_{0}$, a normal distribution with mean zero and a covariance matrix whose $(i-j)$ th entry is $\left\langle h_{i}, h_{j}\right\rangle_{H}$, where $\langle,\rangle_{H}$ is the inner product of $H$. See Millar (1979) for a bit more detail on this point.

Here is an important example of statistical experiments $E^{n}$ converging to a Gaussian shift family.

(4.8) EXAMPLE. Fix $F, f,(\tau, H, B)$ of Example (4.6). For $h \in H$, define $f(h ; x)=$ $f(x)[1+h(x)]$. Denote by $F(h ; t)$ the cdf of $f(h ; x)$; note that $F h=F+\tau h$. Let $P_{h}^{n}$ be the $n$-fold product measure of $f\left(n^{-1 / 2} h ; x\right)$. Let $H_{0}$ be the (dense) subset of $h \in H$ such that $f\left(n^{-1 / 2} h\right)$ is a probability for all sufficiently large $n$. Then $\left\{P_{h}^{n}: h \in H_{0}\right\}$ converges to $\left\{Q_{h}, h \in H_{0}\right\}$, the Gaussian shift experiment of $(\tau, H, B)$. This may be checked by the method of Lemma (4.1), for example.

We are now ready to describe the required special case of the asymptotic minimax theorem. Let $(\tau, H, B)$ be an abstract Wiener space, with $\left\{Q_{h}\right\}$ its standard Gaussian shift experiment. Let $H_{0}$ be a dense subset of $H$, and suppose $E^{n}=\left\{P_{h}^{n}, h \in H_{0}\right\}$ is a sequence of experiments converging to $E=\left\{Q_{h}, h \in H_{0}\right\}$. We shall take $B$ as decision space-i.e., all statistical procedures to be considered below shall be $B$-valued. A nonnegative function $l$ on $B$ is subconvex if it is lower semicontinuous, symmetric $(l(x)=l(-x))$ and $\{x: l(x) \leq c\}$ is convex for every $c>0$. For example, if ||$_{B}$ is the norm of $B$ and $g$ is an increasing function, $l(x)=g\left(|x|_{B}\right)$ is subconvex. A loss function on $B \times H_{0}$ shall be defined by $l(x-\tau h)$ if $x \in B, h \in H$.

(4.9) THEOREM. With the assumptions of the preceding paragraph,

$$
\liminf _{n} \sup _{T_{n} \in H_{0}} \int l\left(T_{n}-\tau h\right) d P_{h}^{n} \geq \int l(x) P_{0}(d x),
$$

where the inf is taken over all B-valued estimators available at time $n$.

Applications to the local asymptotic minimax theory require a slight variant of this result. For each $c$ let $N_{c}$ be a convex subset of $H_{0}, N_{c} \subset N_{c^{\prime}}$ if $c<c^{\prime}$, and $\bigcup N_{0}=H_{0}$. Then, with the assumptions of Theorem (4.9),

$$
\lim _{c \uparrow \infty} \liminf _{n} \sup _{T_{n}} \int l\left(T_{n}-\tau h\right) d P_{h}^{n} \geq \int l(x) P_{0}(d x) .
$$

More abstract and better versions of these results, together with proofs, may be found in Millar (1979) and, of course, Le Cam (1972).

V. A general description of the LAM structure. Estimators of the type introduced in $\S 2$ typically have a strong optimality property. This section gives a general description that applies to all of the applications in this paper. 
A description of this property involves several ingredients. First, suppose:

(5.1) $(\tau, H, B)$ is an abstract Wiener space with $\left\{Q_{h}\right\}$ its Gaussian shift family.

(5.2) For a dense set $H_{0} \subset H$, there are statistical experiments $\left\{P_{h}^{n}: h \in H_{0}\right\}$ converging to $\left\{Q_{h}, h \in H_{0}\right\}$.

For each $P_{h}^{n}$, let

(5.3) $\left\{\xi_{n}\left(\theta, P_{h}^{n}\right): \theta \in \Theta\right\}$ be a stochastic process with values in $B_{2}$, a Hilbert space.

In many applications, $B=B_{2}$. Let

(5.4) $\theta_{n, h}$ be the point in $\theta$ that achieves

$$
\inf _{\theta}\left|\xi_{n}\left(\theta, P_{h}^{n}\right)\right|_{B_{2}}=\left|\xi_{n}\left(\theta_{n h}, P_{h}^{n}\right)\right|_{B_{2}} .
$$

Of course, $\theta_{n h}$ typically will be random. The statistical problem will be to estimate $\theta_{n h}$ when the measure $P_{h}^{n}, h \in H_{0}$, is "unknown". More precisely, let $g$ be an increasing function on $[0, \infty)$; define the loss when the guess $\tilde{\theta}_{n}$ is made and when $P_{h}^{n}$ is 'true' by

$$
g\left(\left|\tilde{\theta}_{n}-\theta_{n h}\right|\right)
$$

where $|\cdot|$ is the norm of $R^{d}$.

The risk of an estimate $\tilde{\theta}_{n}$ is then

$$
\int g\left(\left|\tilde{\theta}_{n}-\theta_{n h}\right|\right) d P_{h}^{n}
$$

Fix now a point $\theta_{0} \in \Theta$. Assume that the processes $\xi_{n}\left(\theta, P_{h}^{n}\right)$ satisfy

$$
\xi_{n}\left(\theta_{0}, P_{h}^{n}\right)=\Psi_{n}+V \circ \tau h+o(1), \quad \forall h \in H_{0},
$$

where

$$
V: B \rightarrow B_{2}
$$

is bounded linear, and $\Psi_{n}$ is a $B_{2}$-valued random variable independent of $h$. In many applications, $B=B_{2}$ and $V$ is the identity.

Assume further that, for each $h, \xi_{n}\left(\theta, P_{h}^{n}\right)$ satisfies the identifiability, differentiability, and boundedness hypotheses of $\S 2$ (under the sequence of measures $\left\{P_{h}^{n}\right\}$ ), with derivative $\eta$ independent of $h$. Let

$$
\pi=\text { projection in } B_{2} \text { to } B_{\eta}
$$

where $B_{\eta}$ is the subspace spanned by the components of $\eta$ (see (2.6)). Here are two examples which illustrate the foregoing set-up-in particular, (5.7).

(5.10) EXAMPLE (CLASSIC CASE). Let $\left\{P_{\theta}, \theta \in \theta\right\}$ be a parametric family of distributions on the line; assume each $P_{\theta}$ has density $f(\theta ; x)$ w.r.t. some sigma-finite measure $m$. Fix $\theta_{0} \in \Theta$ and define densities $f(h ; x)$ by $f(h ; x)=f\left(\theta_{0} ; x\right)[1+h(x)]$, where $h \in H_{0}$ is given in (4.6), (4.8) (with $F=P_{\theta_{0}}$ there). Define $P_{h}^{n}$ as in (4.8) (it is the product of $\left.f\left(h n^{-1 / 2}\right)\right)$ and $(\tau, H, B)$ as in (4.6). If $F(h ; t), F(\theta ; t)$ denote cdf's of $f(h ; \cdot), f(\theta ; \cdot)$, define

$$
\xi_{n}\left(\theta, P_{h}^{n}\right)=n^{1 / 2}\left[F\left(n^{-1 / 2} h\right)-F\left(\theta_{0}+n^{-1 / 2} \theta\right)\right]
$$

(the minor abuse of notation here and elsewhere will cause no confusion.) Since

$$
F\left(n^{-1 / 2} h\right)=F\left(\theta_{0}\right)+n^{-1 / 2} \tau h,
$$


$\xi_{n}\left(\theta, P_{h}^{n}\right)=\xi_{n}\left(\theta, P_{0}^{n}\right)+\tau h$ for every $\theta$, so (5.8) holds with $V$ the identity. This example is a special case of the situation in $\S 6$, where $V$, in general, is not the identity; the fact that the hypotheses of differentiability, etc., hold for $\xi_{n}\left(\theta, P_{h}^{n}\right)$, as defined here, is treated in greater generality in $\S 6$. But this example furnishes a canonical example of a $\xi_{n}\left(\theta, P_{h}^{n}\right)$-process (the measures $P_{h}^{n}$ are relevant for checking out the basic hypotheses of differentiability, boundedness, etc., as required for (5.12)).

If $\tilde{\theta}_{n}$ is an estimate of $\theta$ here, let $\tilde{\theta}_{1 n}=\theta_{0}+n^{-1 / 2} \tilde{\theta}_{n}$; define $\theta_{1 n h}$ similarly. Typically one wants to estimate $\theta_{1 n h}$ instead; in this case the loss function has the more familiar form

$$
g\left(n^{1 / 2}\left|\tilde{\theta}_{1 n}-\theta_{1 n h}\right|\right)=g\left(\left|\tilde{\theta}_{n}-\theta_{n h}\right|\right)
$$

See the end of this section for a general formulation under " $n^{1 / 2}$-scaling".

(5.11) EXAMPLE (SIMPLE REGRESSION). (Refer to $§ 1$ for a brief description of the relevant model.) Fix a distribution $F$ on the line and define $(\tau, H, B)$ as in (4.6). Let $\left\{d_{n i}\right\}$ satisfy $\sum_{1}^{n} d_{n i}^{2}=1$. Let $P_{h}^{n}$ be the product measure $\prod_{1}^{n} f\left(d_{n i} h ; x_{i}\right)$, where $f(h ; \cdot)$ is defined in $(4.8)$. For real $\theta$ let

$$
\xi_{n}\left(\theta, P_{h}^{n}\right)(t)=\sum d_{n i}\left[I\left\{X_{n i} \leq t+c_{n i} \theta\right\}-F\left(d_{n i} h ; t\right)\right],
$$

where $F(h ; \cdot)$ is the cdf of $f(h ; \cdot)$. Since $F\left(d_{n i} h\right)=F(o)+d_{n i} \tau h$ and $\sum d_{n i}^{2}=1$, $\xi_{n}$ here satisfies (5.8). Again, the form of $P_{h}^{n}$ is used to check the differentiability, et al.

We are now ready for the basic LAM lower bound. Define $\pi$ by (2.6). Let $N_{c}$ be any convex subsets of $H_{0}, N_{c} \uparrow H_{0}$. The sets $N_{c}$ are called neighborhoods.

(5.12) THEOREM (LAM LOWER BOUND).

$$
\begin{aligned}
\lim _{c \uparrow \infty} \liminf _{n} \sup _{\tilde{\theta}_{n}} \int g\left(\delta_{n}\left|\tilde{\theta}_{n}-\theta_{n h}\right|\right) d P_{h}^{n} \\
\geq \int g\left(\left|T^{-1} \circ \pi \circ V x\right| Q_{0}(d x)=E g(Z) .\right.
\end{aligned}
$$

Here the infinium is computed over all estimates $\tilde{\theta}_{n}$ of $\theta_{n h}$ and

$$
Z=T^{-1} \circ \pi \circ V \circ X
$$

where $X$ is a $B$-valued random variable with distribution $Q_{0} ; Q_{0}$ was given in (5.1). Of course $Z$ has a normal distribution on $R^{d}$ with mean 0 ; its covariance can be calculated using $(2.20)$.

(5.12) gives an asymptotic lower bound to the minimax risk (when it is defined by (5.6)). The chief difficulty in applying this result is to choose $(\tau, H, B)$ and $\left\{P_{h}^{n}\right\}$ properly so that the bound obtained for that choice is actually attained by some estimator $\hat{\theta}_{n}$. Many examples of such choices are given in the remainder of the paper.

PROOF. It will be enough to give the proof for $g$ bounded, uniformly continuous. Because of (2.11) and (5.8),

$$
\delta_{n}\left(\theta_{n h}-\theta_{0}\right) \doteq-T^{-1} \circ \pi \xi_{n}\left(\theta_{0}, P_{h}^{n}\right) \doteq-T^{-1} \circ \pi\left[\Psi_{n}+V \tau h\right] .
$$

Therefore, the loss at time $n$ is

$$
g\left(\delta_{n}\left|\tilde{\theta}_{n}-\theta_{n h}\right|\right)=g\left(\left|\tilde{\theta}_{n 1}-T^{-1} \circ \pi \circ V \tau h\right|\right),
$$


where $\tilde{\theta}_{n 1}$ is just the "estimate"

$$
\theta_{n 1}=-\delta_{n}\left(\tilde{\theta}_{n}-\theta_{0}\right)-T^{-1} \circ \pi \Psi_{n} .
$$

Let $\phi$ be the map of $B \rightarrow R^{d}$ given by $\phi=T^{-1} \circ \pi \circ V$. Define $\tau_{1}: H \rightarrow R^{d}$ by $\tau_{1} h=$ $\phi \circ \tau h$. Let $B_{1}=\tau_{1} H$; let $H_{1}$ be any subspace of $H$ such that $\tau_{1}: H_{1} \rightarrow B_{1}$ is one-toone. Then $\left(\tau_{1}, H_{1}, B_{1}\right)$ is an abstract Wiener space, and, if $Q_{h}^{0}$ denotes its Gaussian shift experiment, then $Q_{0}^{0}$ is the distribution of $\phi(Z)$, where $Z$ has distribution $Q_{0}$. Since the experiments $\left\{Q_{h}^{0}, h \in H_{1}\right\},\left\{Q_{h}, h \in H_{1}\right\}$ are equivalent, $\left\{Q_{h}^{n}, h \in H_{1}\right\}$ converges to $\left\{Q_{h}^{0}, h \in H_{1}\right\}$. Therefore, by (5.14),

$$
\begin{aligned}
\inf _{\tilde{\theta}_{n}} \sup _{h \in N_{c}} \int g\left(\delta_{n}\left|\tilde{\theta}_{n}-\theta_{n h}\right|\right) d P_{h}^{n} \\
\geq \inf _{\tilde{\theta}_{n}} \sup _{h \in N_{c} \cap H_{1}} \int g\left(\left|\tilde{\theta}_{n}-\tau_{1} h\right|\right) d P_{h}^{n},
\end{aligned}
$$

where the second inf is over all estimates with values in $B_{1}$ (since $\tau_{1} h \in B_{1}$, one can only do worse by taking estimates $\tilde{\theta}_{n}$ with values in $R^{d}-B_{1}$, by the projection theorem in $R^{d}$; the first inf in (5.15) is over all $R^{d}$-valued estimates). Since $l(x) \equiv$ $g(|x|)$ is subconvex on $B_{1}$, the asymptotic minimax theorem of $(4.10)$ shows the last expression in (5.15) is bigger than $\int g(|x|) d Q_{0}^{0}$, on letting $c \uparrow \infty$. Since $Q_{0}^{0}$ is the distribution of $\phi(Z)$, where $Z$ has distribution $Q_{0}$, the result follows. Q.E.D. if

(5.16) DEFINITION. A sequence of estimators $\hat{\theta}_{n}$ of $\left\{\theta_{n h}, h \in H_{0}\right\}$ is called LAM

$$
\lim _{c} \lim _{n} \sup _{h \in N_{c}} \int g\left(\delta_{n}\left|\hat{\theta}_{n}-\theta_{n h}\right|\right) d P_{h}^{n}=E g(Z) .
$$

A priori this definition depends on $g$ and $\left\{N_{c}\right\}$; however, as a practical matter, in most applications the LAM property holds for a given $\hat{\theta}_{n}$ over a large variety of $g,\left\{N_{c}\right\}$.

(5.17) METhOdOLOGiCAL REMARK. In all applications of this paper, a selected sequence $\hat{\theta}_{n}$ of estimators is shown to be LAM by means of the following device. Pick $c$. Let $h_{n}$ be an arbitrary sequence in $N_{c}$. One then shows that, under $P_{h_{n}}^{n}$, $\delta_{n}\left(\hat{\theta}-\theta_{n h_{n}}\right)$ converges in distribution to $Z$, where $Z$ was defined in (5.13). If, say, $g$ is bounded, then this will indeed prove $\hat{\theta}_{n}$ LAM, since $h_{n}$ can be chosen to satisfy

$$
\sup _{h \in N_{c}} \int g\left(\delta_{n}\left|\hat{\theta}_{n}-\theta_{n h}\right|\right) d P_{h}^{n}=\int g\left(\delta_{n}\left|\hat{\theta}-\theta_{n h_{n}}\right|\right) d P_{h_{n}}^{n}
$$

(or to come within $n^{-1}$ of satisfying this relation).

(5.18) Reformulation for $n^{1 / 2}$ scaling. For convenience in later applications we write out the LAM result for the situation discussed in §3. Suppose (5.1)-(5.3) hold, and define $\theta_{n h}$ by (5.4). Replace hypothesis (5.7) by the assumption

$$
n^{1 / 2} \xi_{n}\left(\theta_{0}, P_{h}^{n}\right)=\Psi_{n}+V \circ \tau h+o(1) .
$$

Assume further that the processes $\xi_{n}\left(\theta, P_{h}^{n}\right)$ satisfy the identifiability, differentiability, and boundedness assumptions of $\S 3$, with nonsingular derivative $T$ independent of $h$. Under these conditions one has the following variant of the LAM result. 
(5.20) THEOREM.

$$
\begin{aligned}
& \lim _{c \uparrow \infty} \liminf _{n} \sup _{\tilde{\theta}_{n}} \int g\left(n^{1 / 2}\left|\tilde{\theta}_{n}-\theta_{n h}\right|\right) d P_{h}^{n} \\
& \geq \int g\left(\left|T^{-1} \circ \pi \circ V x\right|\right) Q_{0}(d x)=E g(Z),
\end{aligned}
$$

where $Z=T^{-1} \circ \pi \circ V \circ X$ and $X$ is a $B$-valued random variable with distribution $Q_{0}$.

One naturally defines, in the present situation, a sequence of estimators $\hat{\theta}_{n}$ to be LAM if

$$
\lim _{c} \lim _{n} \sup _{h \in N_{c}} \int g\left(n^{1 / 2}\left|\hat{\theta}_{n}-\theta_{n h}\right|\right) d P_{h}^{n}=E g(Z) .
$$

Again, in all applications, a particular sequence $\hat{\theta}_{n}$ is proved to be LAM by showing that, under $P_{h_{n}}^{n}, n^{1 / 2}\left(\hat{\theta}_{n}-\theta_{n h_{n}}\right)$ converges to $Z$ whenever $h_{n}$ is a sequence in some fixed $N_{c}$. Theorem (5.20) is proved by the method suggested in $\S 3$; see also Example (5.10).

VI. Weighted Cramer-von Mises estimation. The LAM results of this section were developed by Millar (1981). We therefore content ourselves in this section to showing how this particular example fits into the general framework of $\S \S 3,5$. For another investigation of this particular class of estimators, see Parr-de Wet and Parr-Schucany.

Let $X_{1}, \ldots, X_{n}$ be i.i.d. random variables. Let $\left\{P_{\theta}, \theta \in \Theta\right\}$ be a parametric family of measures on the line, where $\Theta$ is an open subset of $R^{d}$. Let

$$
F(\theta ; t)=P_{\theta}\{(-\infty, t]\}
$$

be the cumulative distribution function of $P_{\theta}$, and let

$$
\hat{F}_{n}(t)=n^{-1} \sum_{1}^{n} I_{(-\infty, t)}\left(X_{i}\right)
$$

be the empirical cdf.

Let $\mu$ be a measure on the line and ||$_{\mu}$ the $L^{2}(\mu)$ norm. For each $\theta$, let $q_{\theta}(\cdot) \equiv$ $q(\theta ; \cdot)$ be a real function defined on the line; $q$ is often called a weight function.

For real functions $a(\cdot)$ let

$$
|a|_{\theta}^{2}=\int a^{2}(s) q^{2}(\theta ; s) \mu(d s)=\left|a q_{\theta}\right|_{\mu}^{2} .
$$

The classic Cramer-von Mises minimum distance estimate (with weight function $q$ ) is defined by

$$
\inf _{\theta}\left|\hat{F}_{n}-F_{\theta}\right|_{\theta}=\left|\hat{F}_{n}-F_{\hat{\theta}_{n}}\right|_{\hat{\theta}_{n}}
$$

For convenience, $\hat{\theta}_{n}$ will be referred to as CVMMDE. The choice $q(\theta ; t) \equiv 1$ gives the "classic" minimum distance estimator mentioned in (1.1).

The goal of this section is to establish, in an appropriate framework, the local asymptotic optimality of the estimator $\hat{\theta}_{n}$.

The first step is to place the problem into the general framework of $\S \S 3,5$. To this end, fix a point $\theta_{0} \in \Theta$; we shall establish a LAM property about "neighborhoods" 
of $P_{\theta_{0}}$. Assume first that there is a sigma-finite measure $m$ such that each $P_{\theta}$ has density $f(\theta, \cdot)$ w.r.t. $m$. Define $H$, the Hilbert space of real functions $h$, by

$$
\begin{aligned}
H=\{h: & \int h(s)^{2} f\left(\theta_{0} ; s\right) m(d s)<\infty \\
& \left.\int h(s) f\left(\theta_{0} ; s\right) m(d s)=0, \text { support } h \subset \operatorname{support} f\left(\theta_{0}, \cdot\right)\right\} .
\end{aligned}
$$

Define, in addition,

$$
\begin{aligned}
f(h ; t) & =f\left(\theta_{0} ; t\right)[1+h(t)], \\
H_{0} & =\left\{h \in H: f\left(h n^{-1 / 2} ; \cdot\right) \geq 0 \text { for all large } n\right\}, \\
F(h ; \cdot) & =c d f \text { of } f(h ; \cdot) .
\end{aligned}
$$

Then $H_{0}$ is a dense convex subset of $H$ and, if $h \in H_{0}, f\left(h n^{-1 / 2}\right)$ is a probability density, at least for all large $n$. Define the product measure

$$
P_{h}^{n}(d x)=\prod_{1}^{n} f\left(h n^{-1 / 2} ; x_{i}\right) d x, \quad x=\left(x_{1}, \ldots, x_{n}\right) .
$$

Using the method suggested in (4.1), one easily sees that the experiments $\left\{P_{h}^{n}, h \in\right.$ $\left.H_{0}\right\}$ converge to an experiment $\left\{Q_{h}, h \in H_{0}\right\}$. Indeed, let $H$ be defined as above, and

$$
\tau h(t)=\int^{t} h(s) f\left(\theta_{0} ; s\right) m(d s) .
$$

If $\mu$ is finite (which we assume from now on; variants for sigma-finite $\mu$ are not difficult, but take a fair amount of space to discuss), then

$$
\tau h \subset L^{2}(\mu) \text {. }
$$

Define the Hilbert space $B$ by

$$
B=\operatorname{closure}\{\tau h: h \in H\} .
$$

Then $(\tau, H, B)$ is an abstract Wiener space (cf. $\S 4)$ and, if $\left\{Q_{h}, h \in H\right\}$ is its canonical normal shift family,

$$
Q_{0} \text { is the distribution of } W_{F}^{0} \text {, }
$$

where $W_{F}^{0}(t)=W^{0}\left(F\left(\theta_{0} ; t\right)\right), t \in R^{1}$, and $W^{0}$ is the usual Brownian bridge on $[0,1]$. As usual, $Q_{h}(A)=Q_{0}(A-\tau h), A=$ Borel set of $L^{2}(\mu)$. The convergence of $\left\{P_{h}^{n}, h \in H_{0}\right\}$ to $\left\{Q_{h}, h \in H_{0}\right\}$ was discussed in $\S 4$.

To state the basic LAM result, make the following assumptions and definitions. Assume that the maps

$$
\theta \rightarrow q_{\theta}, \quad \theta \rightarrow F_{\theta} q_{\theta}, \quad \theta \rightarrow F_{\theta}
$$

of $\Theta$ to $L^{2}(\mu)$ are Fréchet differentiable at $\theta_{0}$ with derivatives $\eta_{1}, \eta_{2}, F_{\theta_{0}}^{\prime}$, respectively. Denote by $\eta$ the element of $L^{2}(\mu)$ given by

$$
\eta=\eta_{1} F_{\theta_{0}}-\eta_{2}=-F_{\theta_{0}}^{\prime} q_{\theta_{0}} .
$$

The derivative $\eta$ is assumed nonsingular. Assume further that

$$
\text { if } \theta_{n} \text { is any sequence in } \Theta \text { such that }\left|F_{\theta_{n}}-F_{\theta_{0}}\right|_{\theta_{n}} \rightarrow 0 \text {, then } \theta_{n} \rightarrow \theta_{0} \text {. }
$$


Define $V$, multiplication operator by

$$
V z=q_{\theta_{0}} \times z, \quad z \in L^{2}(\mu) .
$$

Let $\pi$ be projection to the subspace of $L^{2}$ spanned by the components of $\eta$; define $T$ as in (2.10). Define

$$
N_{c}=\left\{h \in H_{0}:|h| \leq c\right\},
$$

and let $g$, bounded, be defined as in $\S 5$. Define $\theta_{n h}$ by

$$
\inf _{\theta}\left|F\left(n^{-1 / 2} h\right)-F(\theta)\right|_{\theta}=\left|F\left(n^{-1 / 2} h\right)-F\left(\theta_{n h}\right)\right|_{\theta_{n h}}
$$

for $h \in H_{0}$. Recall $\hat{\theta}_{n}$, the "CVMMD" estimator of (6.4).

(6.17) THEOREM (LAM PROPERTY OF THE CVMMD ESTIMATE).

$$
\begin{aligned}
\lim _{c \uparrow \infty} \liminf _{n} \sup _{\tilde{\theta}_{n}} \int g\left(n^{1 / 2}\left|\tilde{\theta}_{n}-\theta_{n h}\right|\right) d P_{h}^{n} \\
\quad=\int g\left(T^{-1} \circ \pi \circ V \circ z\right) Q_{0}(d z) \\
\quad=\lim _{c \uparrow \infty} \lim _{n} \sup _{h \in N_{c}} \int g\left(n^{1 / 2}\left|\hat{\theta}_{n}-\theta_{n h}\right|\right) d P_{h}^{n} .
\end{aligned}
$$

Here the inf is over all estimates $\tilde{\theta}_{n}$ of $\theta$; see $\S 7$ for statistical interpretation of this result.

(6.18) REMARK. If the first equality is replaced by $\geq$, then the first half of the result remains valid if $g$ is only increasing (boundedness of $g$ is not necessary to establish the second expresion as the LAM lower bound).

(6.19) REMARK. The question here is really the estimation of a functional $\theta(\cdot)$ defined on distributions $G$ by $G \rightarrow \theta(G)$,

$$
\inf _{\theta}\left|G-F_{\theta}\right|_{\theta}=\left|G-F_{\theta(G)}\right|_{\theta(G)} .
$$

The functional is considered here only on the measures $F\left(n^{-1 / 2} h, d x\right)$ and $f\left(h n^{-1 / 2} ; x\right) d x$, so $\theta\left(F\left(n^{-1 / 2} h\right)\right)=\theta_{n h}$; actually it can be considered on many more $G$-see $\S 7$ for some of them, and for reasons for such an extension. Part of the proof of (6.16) centers on showing that the map $F_{n^{-1 / 2} h} \rightarrow \theta\left(F_{n^{-1 / 2} h}\right)$ is "differentiable" in the sense that $\left[\theta\left(F_{n^{-1 / 2} h}\right)-\theta\left(F_{0}\right)\right] n^{1 / 2}$ is, essentially, a linear function of $h$, as $n \rightarrow \infty$. For showing this, the basic result of $\S 3$ is the key. Evidently, there is an abstract LAM result that can be formulated for a wide class of "differentiable functions", not just those of the minimum distance type.

PROOF. Let us begin by proving the last equality in the theorem. For this, we shall use the method suggested in (5.17). Fix $c$ and let $h_{n}$ be an arbitrary sequence in $N_{c}$; we must show that $n^{1 / 2}\left(\hat{\theta}_{n}-\theta_{n h_{n}}\right)$ converges, under $P_{h n}^{n}$, to $T^{-1} \circ \pi \circ V \circ Z$, where $Z$ has distribution $Q_{0}$. Define processes

$$
\xi_{n}^{1}\left(\theta, P_{h n}^{n}\right)=\left(F\left(n^{-1 / 2} h_{n}\right)-F_{\theta}\right) q_{\theta}, \quad \xi_{n}^{2}(\theta)=\left(\hat{F}_{n}-F_{\theta}\right) q_{\theta} .
$$

Because

$$
F\left(n^{-1 / 2} h\right)=F_{\theta_{0}}+\tau h n^{-1 / 2},
$$

and because, under $P_{h n}^{n},\left[\hat{F}_{n}-F\left(n^{-1 / 2} h n\right)\right] n^{1 / 2}$ converges in $L^{2}(\mu)$ to $W_{F}$ (an application of the CLT for Hilbert space-see Parthasarathy (1967)), it is easy to see 
that $\xi_{h}^{1}, \xi_{h}^{2}$ satisfy the hypothesis of boundedness as defined in $\S 3$ (under the sequence of measures $\left.P_{h n}^{n_{n}}\right)$. The hypothesis of convergence need NOT be satisfied here. Simple calculations show that (6.11), (6.13) imply, respectively, the hypotheses of differentiability, identifiability for both processes $\xi_{n}^{1}, \xi_{n}^{2}$; the derivative in each case, of course, is $\eta$. For this one needs to know, e.g., that $\hat{F}_{n}, F\left(n^{-1 / 2} h\right)$ both converge, under $P_{h_{n}}^{n}$, in $L^{2}(\mu)$ to $F_{\theta_{0}}$. Therefore, the main result of $\S 3$ yields

$$
\begin{aligned}
n^{1 / 2}\left(\hat{\theta}_{n}-\theta_{0}\right) & =-T^{-1} \circ \pi \circ \xi_{n}^{2}\left(\theta_{0}\right)+o(1), \\
n^{1 / 2}\left(\theta_{n h_{n}}-\theta_{0}\right) & =-T^{-1} \circ \pi \circ \xi_{n}^{1}\left(\theta_{0}\right)+o(1),
\end{aligned}
$$

and so

$$
n^{1 / 2}\left(\hat{\theta}_{n}-\theta_{n h_{n}}\right)=-T^{-1} \circ \pi \circ\left(\xi_{n}^{2}\left(\theta_{0}\right)-\xi_{n}^{1}\left(\theta_{0}\right)\right)+o(1)
$$

Since

$$
\begin{aligned}
\xi_{n}^{2}\left(\theta_{0}\right)-\xi_{n}^{1}\left(\theta_{0}\right) & =n^{1 / 2}\left[\hat{F}_{n}-F\left(n^{-1 / 2} h\right)\right] q_{\theta_{0}} \\
& \Rightarrow V \circ W_{F} \quad \text { under } P_{h_{n}}^{n},
\end{aligned}
$$

the second equality of (6.17) follows from (6.18) and the boundedness of $g$.

Let us turn now to proving that the first expression in (6.16) is at least as big as the second expression there. To do this define

$$
\xi_{n}\left(\theta, P_{h}^{n}\right)=\left(F\left(n^{-1 / 2} h\right)-F_{\theta}\right) q_{\theta} .
$$

Since $F\left(n^{-1 / 2} h\right)=F_{\theta_{0}}+n^{-1 / 2} \tau h$, we see that

$$
n^{1 / 2} \xi_{n}\left(\theta_{0}, P_{h}^{n}\right)=n^{1 / 2} \xi_{n}\left(\theta_{0}, P_{0}^{n}\right)+V \circ \tau h,
$$

so that hypothesis (5.20) is satisfied. As in the first part of the proof, $\xi\left(\theta, P_{h}^{n}\right)$ satisfies the hypotheses of boundedness and differentiability-because of (6.11), (6.13). Therefore, the desired result is immediate from (5.21). Q.E.D.

VII. Comments on the statistical meaning of the result of $\S 6$. In this section we comment briefly on the statistical meaning of the setup advanced in $\S 6$; with minor changes, these comments carry over to all of the applications.

The observations $X_{1}, \ldots, X_{n}$ are i.i.d., but it is presumed that the common distribution $F$ may be different from the distributions $\left\{P_{\theta}, \theta \in \Theta\right\}$ specified by the theoretical model. That is, the model does not precisely describe the data distribution, but it is presumed that it is not too unreasonable. There are a number of reasons why one might not have a parametric model that precisely describes the data. One possibility is that the observations have been subject to "data contamination" (roundoff errors, clerical errors, etc.). Another possibility is that the "true" model governing the $X_{i}$ is so complicated (e.g., has inconveniently large dimension) that one would like to use a "simpler" model to fit the data as best one can, the idea being that one might not easily grasp the basic shape of the data if there are several million parameters.

Given that the data has a distribution that may differ from the model $\left\{P_{\theta}, \theta \in\right.$ $\boldsymbol{\theta}$, the first step is to specify what the possible data distributions might be. For each fixed $\theta_{0} \in \Theta$, these are, in the model of $\S 6$, given by $\left\{F\left(n^{-1 / 2} h\right): h \in N_{c}\right\}$, where $c$ is an arbitrary fixed number. It is not difficult to see that this collection of data distributions can be augmented considerably. For example, the collection of 
data distributions just mentioned could be replaced by the collection of all cdf's $F$ such that $\sup _{t}\left|F(t)-P_{\theta_{0}}(t)\right| \leq c n^{-1 / 2}$ (where $P_{\theta_{0}}(t)=\operatorname{cdf}$ of $P_{\theta_{0}}$ ), and the basic theorem of $\S 6$ will continue to hold. This allows an extremely large collection of possible data distributions; other minimum distance methods (cf. $\S \S 13,12)$ will admit only relatively small collections of data distributions, a feature that sometimes is a severe disadvantage.

Having specificed the model $\left\{P_{\theta}, \theta \in \Theta\right\}$ and the possible data distributions, the task is to 'fit the model to the data as best one can'. As discussed in $\S 1$, there are a great many possibilities, depending on what the statistical goals are. The criterion for 'best fit' adopted in $\S 6$ is given by $(6.16),(6.20)$; for this to be acceptable it is more or less tacit that the statistical goals include the estimation of a number of probabilities; if one wanted to estimate moments as well, a somewhat different notion of best fit should be adopted (see $\S 13$, Example 4 for one possibility); and if one wanted quantiles estimated too, then probably yet another framework should be set up (cf. $\S 13$, Example 6(c)). In view of the last sentence of the preceding paragraph, the notion of best fit to be adopted will also have to take into account the kind of data distributions you believe are present: if $G$ is a possible data distribution you evidently at least have to be able to say what the 'distance' between $G$ and $\left\{P_{\theta}\right\}$ is. If the class of data distributions is believed to be rather broad, the approach of $\S 6$ is appropriate; if you are sure the possible data distributions form a fairly small class, then you could use some of the other methods in $\S 13$.

Having specified the possible data distributions and an appropriate notion of 'best fit' (in the case under discussion it is specified by (6.20)), we wish to find the point $\theta$ that 'fits' $P_{\theta}$ as close as possible to the actual (unknown) data distribution $G$. That is, we wish to estimate $\theta(G)$, defined in (6.19). The result (6.17) is an optimality theorem elaborated in just such a framework. Such LAM results have a long history, going back to classical parametric estimation; discussion of their statistical importance should be unnecessary at this point in time (see Hajek (1972), Le Cam (1972), Beran (1981), Millar (1981) for original or recent developments). Let it suffice to say, the intent of LAM theorems is to ensure at least a desirable stability of estimators in the convergence to their asymptotic limit.

Questions can, of course, be raised about the choice of the particular framework of $\S 6$ (and for all of the other applications). Fortunately, the setup of $\S 6$ has been heavily studied. A certain amount of confidence in the excellence of the procedures advanced there derives from the fact that they actually work well in practice. See Parr-Schucany for some empirical studies; see Millar (1981) for arguments (based on (2.12), (2.16)) showing their asymptotic equivalence to other procedures that have been advanced. That these procedures should behave decently is demonstrated theoretically in the proof of (6.17), where the crux of the matter was to show convergence to the asymptotic limit, that is uniform over the large neighborhoods $N_{c}$. Finally, if the possible data distributions are truly broad, as assumed in $\S 6$, the LAM framework nicely rules out certain 'unstable' estimators. For example, if $P_{\theta}$ were $N(\theta, 1)$ one could use $\bar{X}$, the sample mean, to estimate the $\theta$ that 'fits best'; it is easy to see this will not be LAM, since it is always possible to find neighboring data distributions $G,\left|G-P_{\theta_{0}}\right| \leq n^{-1 / 2}, G$ with no mean; this will force $\bar{X}$ to be extremely unstable asymptotically (see Millar (1981) for the precise calculation). Evidently, the entire framework of $\S 6$ could be cast in terms of robustness; but this 
was done already in Millar (1981); robustness terminology can also be appled to some of the other developments; we leave this to the reader.

VIII. Minimum $\chi^{2}$ estimates. The estimation method of this section is a simple variant of those of $\S 6$; it is worth singling out because minimum $\chi^{2}$ methods have enormous importance in applied statistics.

Let $\left\{P_{\theta}, \theta \in \Theta\right\}$ be a family of probabilities concentrated on the integers $1, \ldots, d$ and indexed by an open subset $\theta \subset R^{s}, s \leq d$. Denote by

$$
\begin{aligned}
F_{\theta}(t) & =F(\theta ; t) \text { the cdf of } P_{\theta}, \\
f(\theta ; i) & =f_{i}(\theta)=\text { mass assigned to }\{i\} \text { by } P_{\theta}, \\
f(\theta) & =f(\theta ; \cdot) \text { the vector }(f(\theta ; 1), \ldots, f(\theta ; d)) \in R^{d} .
\end{aligned}
$$

If $G$ is any probability on the integers $1, \ldots, d$ with $g_{i}=G(\{i\})$, define the minimum $\chi^{2}$ functional $\theta(G)$ by

$$
\inf _{\theta} \sum \frac{\left[g_{i}-f_{i}(\theta)\right]^{2}}{f_{c}(\theta)}=\sum \frac{\left[g_{i}-f_{i}(\theta(G))\right]^{2}}{f_{i}(\theta(G))} .
$$

As in other examples, some tedium can be avoided if it is assumed that this inf is achieved at some point; otherwise the usual trivial device needs to be employed (take any $\hat{\theta}(G)$ that comes within $n^{-2}$ of achieving the inf). Define, for any $x=$ $\left(x_{1}, \ldots, x_{d}\right) \in R^{d}$

$$
|x|_{\theta}^{2}=\sum \frac{x_{i}^{2}}{f_{i}(\theta)} .
$$

Then if $g$ is the vector $\left(g_{1}, \ldots, g_{d}\right), \theta(G)$ satisfies

$$
\inf _{\theta}\left|g-f_{\theta}\right|_{\theta}=\left|g-f_{\theta(G)}\right|_{\theta(G)},
$$

a formulation which emphasizes the similarity of $\min \chi^{2}$ methods to minimum distance Cramer-von Mises methods of $\S 6$.

Assume that $X_{1}, \ldots, X_{n}$ are i.i.d. random variables with values in $(1, \ldots, d)$. Let $\hat{F}_{n}$ be the empirical cdf and let

$$
\hat{f}(i)=\hat{F}_{n}(\{i\})
$$

the mass assigned to $\{i\}$ by $\hat{F}_{n}$. The $\min \chi^{2}$ estimate of $\theta$ is

$$
\theta\left(\hat{F}_{n}\right) \text {. }
$$

The goal of this section is to show that $\theta\left(\hat{F}_{n}\right)$ is asymptotically normal and LAM; normality is well known.

To describe these results, fix $\theta_{0} \in \Theta$. Define

$$
H=\left\{h \in R^{d}: \int h d F_{\theta_{0}}=\sum h_{i} f_{c}\left(\theta_{0}\right)=0\right\} .
$$

For $h \in H$, define probabilities $F(h)$ on $\{1, \ldots, d\}$ by specifying that the mass assigned to $\{i\}$ by $F(h)$ shall be $f(h ; i)=f_{i}(h)$, defined by

$$
f(h ; i)=f\left(\theta_{0} ; i\right)\left[1+h_{i}\right]
$$


if $h=\left(h_{1}, \ldots, h_{d}\right)$. See $\S 6$ for an extremely close analogue. Let

$$
P_{h}^{n}, h \in H \text {, be the } n \text {-fold product of } F\left(n^{-1 / 2} h\right) \text {. }
$$

For each $c>0$ define

$$
N_{c}=\left\{h \in H:|h|_{0} \leq c\right\},
$$

where $\left.\right|_{0}$ is the norm of $R^{d}$.

Next let us introduce hypotheses on the family $\left\{F_{\theta}, \theta \in \Theta\right\}$. Assume:

(a) The map $\theta \rightarrow f(\theta)$ of $\theta$ to $R^{d}$ is differentiable.

(b) The derivative is bounded in a neighborhood of $\theta_{0}$.

(c) For each $i, f_{i}(\theta)$ is strictly positive near $\theta_{0}$.

Define vectors $\eta_{1}, \ldots, \eta_{s}, \eta_{i} \in R^{d}$, such that the $j$ th component of $\eta_{i}$ is

$$
\left(\eta_{i}\right)_{j}=\left[f^{1 / 2}(\theta ; j)\right]^{-1}\left[\left(\partial / \partial \theta_{i}\right) f(\theta ; j)_{\theta=\theta_{0}}\right], \quad 1 \leq j \leq d .
$$

Assume the nonsingularity hypothesis

$$
\eta_{1}, \ldots, \eta_{s} \text { are linearly independent. }
$$

Define:

$$
\begin{aligned}
& \pi \text { : projection in } R^{d} \text { to span } \eta_{i} . \\
& T: \text { map of } \Theta \text { to span } \eta_{i} \text { by } T \theta=\langle\theta, \eta\rangle,
\end{aligned}
$$

where $\langle\theta, \eta\rangle=\sum \theta_{i} \eta_{i}$ when $\theta=\left(\theta_{1}, \ldots, \theta_{s}\right)$. Since $\eta$ is nonsingular, $T^{-1}$ exists. Assume, in addition,

$$
\text { if }\left|f\left(\theta_{n}\right)-f\left(\theta_{0}\right)\right|_{\theta_{n}} \rightarrow 0, \text { then } \theta_{n} \rightarrow \theta_{0} .
$$

Define the multiplication operator $V$ on $R^{d}$ by

$$
(V x)_{i}=x_{i} / f^{1 / 2}\left(\theta_{0} ; i\right), \quad x=\left(x_{i}, \ldots, x_{d}\right) .
$$

Let $Q_{0}$ be the normal distribution on $R^{d}$ with mean 0 and covariance matrix $\Sigma$, where the $(i-j)$ th element of $\Sigma$ is

$$
\Sigma_{i j}=\left\{\begin{array}{cc}
-f\left(\theta_{0} ; i\right) f\left(\theta_{0} ; j\right), & i \neq j, \\
f\left(\theta_{0} ; i\right)\left[1-f\left(\theta_{0} ; i\right)\right], & i=j .
\end{array}\right.
$$

Finally, let $l$ be a function on $R^{d}$ of the form

$$
l(x)=g\left(|x|_{0}\right)
$$

where $g$ is a bounded increasing function on $[0, \infty)$. Then the following result establishes the LAM character of the minimum $\chi^{2}$ estimates.

(8.18) THEOREM.

$$
\begin{aligned}
\lim _{c \uparrow \infty} \liminf _{n} \sup _{\tilde{\theta}_{n}} \int l\left(n^{1 / 2}\left(\tilde{\theta}_{n}-\theta_{n h}\right)\right) d P_{h}^{n} \\
=\int l\left(T^{-1} \circ \pi \circ V x\right) Q_{0}(d x) \\
=\lim _{c \uparrow \infty} \lim _{n} \sup _{h \in N_{c}} \int l\left(n^{1 / 2}\left(\hat{\theta}_{n}-\theta_{n h}\right)\right) d P_{h}^{n},
\end{aligned}
$$


where $\theta_{n h}=\theta\left(F\left(n^{-1 / 2} h\right)\right), \hat{\theta}_{n}=\theta\left(\hat{F}_{n}\right)$ and the inf is taken over all estimates $\tilde{\theta}_{n}$ of $\theta$.

Industrious readers may use $(3.8)$ to write out the covariance matrix of $T^{-1} \circ \pi \circ V$.

ProOF. Let us first establish the second equality. For this it is enough (cf. (5.17)) to show that if $h_{n}$ is any sequence in $N_{c}$, then, under $P_{h}^{n}, n^{1 / 2}\left(\hat{\theta}_{n}-\theta_{n h_{n}}\right) \Rightarrow$ $T^{-1} \circ \pi \circ V Z$, where $Z$ has distribution $Q_{0}$. Let $\xi_{n}^{1}$ be the random vector with $i$ th component $\left[\hat{f}_{n}(i)-f_{i}(\theta)\right] / f_{i}^{1 / 2}(\theta)$. Since, under $P_{h}^{n}$,

$$
n^{1 / 2}\left[\hat{f}_{n}-f\left(n^{-1 / 2} h_{n}\right)\right] \Rightarrow Z,
$$

it is clear that $\xi_{n}^{1}$ satisfies the hypothesis of boundedness given in $\S 3$. Hypotheses (8.11), (8.12) and (8.14) ensure differentiability (with nonsingular derivative $\eta$ ) and identifiability respectively (as these are defined in §3). Therefore, by (3.6),

$$
n^{1 / 2}\left(\hat{\theta}_{n}-\theta_{0}\right)=-T^{-1} \circ \pi \circ \xi_{n}^{1}\left(\theta_{0}\right)+o(1) .
$$

Similarly, one shows

$$
n^{1 / 2}\left(\theta_{n h_{n}}-\theta_{0}\right)=-T^{-1} \circ \pi \circ \xi_{n}^{2}\left(\theta_{0}\right)+o(1),
$$

where $\xi_{n}^{2}(\theta)$ is the vector whose $i$ th component is $\left[f\left(n^{-1 / 2} h_{n} ; i\right)-f(\theta ; i)\right] / f^{1 / 2}(\theta ; i)$. Combining (8.20), (8.21) and using the definition of $V$ yields

$$
n^{1 / 2}\left(\hat{\theta}_{n}-\theta_{n h_{n}}\right)=-T^{-1} \circ \pi \circ V \circ\left(\hat{f}_{n}-f\left(n^{-1 / 2} h_{n}\right)\right)+o(1),
$$

which gives the desired conclusion, by (8.19).

To finish the proof of the theorem, it is necessary now to show only that the first expression is at least as big as the second, and, as in other cases, one does not need to assume that $g$ is bounded in order to carry this out. To establish this LAM lower bound, we apply the result of (5.21). For this, define vector processes $\xi_{n}\left(\theta, P_{h}^{n}\right)$ with values in $\{1, \ldots, d\}$ by specifying the $i$ th component of $\xi_{n}\left(\theta, P_{h}^{n}\right)$ to be

$$
\left[f\left(n^{-1 / 2} h ; i\right)-f(\theta ; i)\right] / f^{1 / 2}(\theta ; i) .
$$

Define $\tau_{0}$, the mapping on $H$, by

$$
\left(\tau_{0} h\right)(t)=\int^{t} h(s) F\left(\theta_{0} ; d s\right)
$$

where $h(s)$ is the step function on $[0, d]$ obtained from $\left(h_{1}, \ldots, h_{d}\right)$. Let $Q_{00}$ be the distribution of $W^{0}\left(F\left(\theta_{0} ; t\right)\right)$ and $Q_{0 h}$ its shifts by $\tau_{0}$. Then, as in $\S 6$, the experiments $\left\{P_{h}^{n}, h \in H\right\}$ converge to $\left\{Q_{0 h}\right\}$. The measure $Q_{00}$ may be identified with the distribution of $\left(W^{0}\left(F\left(\theta_{0} ; 1\right)\right), \ldots, W^{0}\left(F\left(\theta_{0} ; d\right)\right)\right)$. Define a mapping $\tau_{1}$ on $R^{d}$ by $\tau_{1} x=\left(x_{1}, x_{2}-x_{1}, \ldots, x_{d}-x_{d-1}\right)$. Let $Q_{0}$ be the image of $Q_{00}$ under $\tau_{1}$; of course, $Q_{0}$ is the same as the measure defined in (8.16). Define $\tau=\tau_{1} \circ \tau$, a mapping on $H$, and let $Q_{h}$ be the shift of $Q_{0}$ by $\tau$. Then since $\tau_{1}$ is one-to-one, $\left\{Q_{n}\right\}$ is equivalent to $\left\{Q_{0 h}\right\}$, so $\left\{P_{h}^{n}\right\}$ converges to $\left\{Q_{h}\right\}$. Moreover,

$$
n^{1 / 2} \xi_{n}\left(\theta_{0}, P_{h}^{n}\right)=V \circ \tau \circ h
$$

since $(\tau h)_{i}=h_{i} f\left(\theta_{0} ; i\right)$. As in the first part of the proof, the processes $\xi_{n}\left(\theta, P_{h}^{n}\right)$ satisfy the hypotheses of convergence, differentiability, and identifiability. The LAM lower bound is now immediate from (5.12). Q.E.D. 
IX. Estimates based on quantile functions. Let $G$ be a probability cdf on the line. Let $\mathcal{Q}$ be the quantile function of $G$ : the function defined on $s \in[0,1]$ by

$$
\mathcal{Q}(G)(s)=\inf \{t: G(t) \geq s\} .
$$

That is, $\mathcal{Q}(G)$ is the usual right continuous inverse of the increasing function $t \rightarrow$ $G(t)$. We shall often write $G^{-1}(s)$ for $\mathcal{Q}(G)(s)$. Let $X_{1}, \ldots, X_{n}$ be real random variables; if $\hat{F}_{n}$ is the empirical cdf then

$$
\mathcal{Q}\left(\hat{F}_{n}\right) \text { is the empirical quantile function. }
$$

Let now $\left\{P_{\theta}, \theta \in \Theta\right\}$ be a fixed family of probabilities on the line indexed by an open subset $\Theta$ of $R^{d}$. Let

$$
F(\theta ; t)=F_{\theta}(t)=\operatorname{cdf} \text { of } P_{\theta} .
$$

The quantile minimum distance functional $\theta(G)$ is defined on cdf's $G$ by

$$
\inf _{\theta}\left|\mathcal{Q}(G)-\mathcal{Q}\left(F_{\theta}\right)\right|=\left|\mathcal{Q}(G)-\mathcal{Q}\left(F_{\theta(G)}\right)\right|,
$$

where || denotes the norm of an appropriate Hilbert space wherein the functions $G^{-1}, F_{\theta}^{-1}$ are alleged to lie. The quantile minimum distance estimate of $\theta$ is

$$
\theta\left(\hat{F}_{n}\right) \equiv \hat{\theta}_{n}
$$

to be denoted QMDE occasionally.

The purpose of this section is to describe the LAM optimality properties of the QMDEs of $\theta$. As in other sections, it is to be understood here that the statistical goal is to obtain the "best fit" of the model to the data, where "best fit" now means matching up the quantile functions as best as one can-a not unreasonable statistical purpose for many investigations.

We shall assume $X_{1}, \ldots, X_{n}$ i.i.d. and, under suitable hypotheses, show $\mathcal{Q}\left(\hat{F}_{n}\right)$ to be asymptotically normal and LAM. Asymptotic normality has been discussed by La Riccia, using a rather less abstract point of view; the LAM aspect appears here for the first time.

Let $0<\alpha<\beta<1$ and let $\mu$ be a finite measure on $[\alpha, \beta]$. To avoid difficulties with the tails of $G^{-1}$, we shall look at $G^{-1}$ only on the interval $[\alpha, \beta]$, and, with this convention, shall regard $G^{-1}$ as an element of $L^{2}(\mu)$. In particular, the minimum distance functional $\theta(G)$ is to be computed using ||, the norm of $L^{2}(\mu)$. Obviously more technically involved set-ups are possible: one can take $\mu$ on $(0,1)$, but then additional hypotheses are necessary so that $G^{-1} \in L^{2}(\mu)$ for the $G$ arising below; in particular, $\mathcal{Q}\left(\hat{F}_{n}\right) \notin L^{2}(\mu)$ for general $\mu$ unless certain conventions are adopted. This superficially greater generality involves no truly new insight, while making the situation relatively messy; therefore, we content ourselves with our present framework. For the parametric family $\left\{P_{\theta}\right\}$, assume:

(9.6) (a) support $F^{-1} \supset[\alpha, \beta]$.

(b) $P_{\theta}$ has density $f_{\theta}$ with respect to Lebesgue measure, $f_{\theta}$ continuous and strictly positive on the interior of its support.

(c) If $F_{\theta_{n}}^{-1}-F_{\theta_{0}}^{-1}$ converges to 0 in $L^{2}(\mu)$, then $\theta_{n} \rightarrow \theta_{0}$.

(d) $F^{-1}(\theta)-F^{-1}\left(\theta_{0}\right)=\left\langle\theta-\theta_{0}, \eta\right\rangle+o(1)$ in $L^{2}(\mu)$, where $\eta=\left(\eta_{1}, \ldots, \eta_{d}\right),\left\{n_{i}\right\}$ a linearly independent set of elements of $L^{2}(\mu)$. 
Under a bit more regularity, and with some effort, hypotheses (c), (d) can be put directly in terms of the cdf's $F_{\theta}$; in some examples (e.g., location model) there is clearly no advantage to doing so. Define $H$ to be the Hilbert space of real functions $h$ on $[0,1]$, such that $\int h^{2}(s) d s<\infty, \int h(s) d s=0$. Let $F_{h}=F(h)$ be the cdf with density

$$
d F_{h}(t)=f\left(\theta_{0} ; t\right)\left[1+h\left(F\left(\theta_{0} ; t\right)\right)\right] .
$$

Define $\tau$ on $H$ by $(\tau h)(t)=\int^{F\left(\theta_{0} ; t\right)} h(s) d s$, so

$$
F_{h}=F_{\theta_{0}}+\tau h \text {. }
$$

Let $B=$ closure of $\tau H$ under sup norm: so $B$ is a subcollection of continuous functions of the form $x\left(F\left(\theta_{0} ; t\right)\right), x \in C[0,1]$. Then $(\tau, H, B)$ is an abstract Wiener space. Let $\left\{Q_{h}\right\}$ be its Gaussian shifts experiment; then $Q_{0}$ is the distribution of $\left\{W^{0}\left(F\left(\theta_{0} ; t\right)\right)\right\}$.

Let $P_{h}^{n}$ be the $n$-fold product of $F\left(n^{-1 / 2} h\right)$; then $\left\{P_{h}^{n}\right\}$ converges to $\left\{Q_{h}\right\}$. Let $B_{2}=L^{2}(\mu)$ and define the map $V: B \rightarrow B_{2}$ by

$$
(V x)(t)=\left[x\left(F^{-1}\left(\theta_{0} ; t\right)\right) / f\left(F_{\theta_{0}}^{-1}(t)\right)\right] I_{[\alpha, \beta]}(t),
$$

where $f(s)=f\left(\theta_{0} ; s\right)$. Define $\pi$, projection in $B_{2}$ to span $\left\{\eta_{i}\right\}$, and define $T$ as usual (cf. (2.10)).

Let $N_{c}=\{h \in H:|h| \leq c, h$ has compact support in $(0,1)$, infinitely differentiable, with bounded derivatives $\}$.

(9.8) THEOREM.

$$
\begin{aligned}
& \lim _{c \uparrow \infty} \liminf _{n} \sup _{\tilde{\theta}_{n}} \int g\left(n^{1 / 2}\left|\tilde{\theta}_{n}-\theta_{n h}\right|\right) d P_{h}^{n} \\
& \quad=\int g\left(\left|T^{-1} \circ \pi \circ V x\right|\right) Q_{0}(d x) \\
& \quad=\lim _{c \uparrow \infty} \lim _{n} \sup _{h \in N_{c}} \int g\left(n^{1 / 2}\left|\hat{\theta}_{n}-\theta_{n h}\right|\right) d P_{h}^{n}
\end{aligned}
$$

where $\hat{\theta}_{n}$ is the $Q M D E$.

Proof. Define

$$
\xi_{n}\left(\theta, P_{h}^{n}\right)(t)=\left[F^{-1}\left(n^{-1 / 2} h ; t\right)-F^{-1}(\theta ; t)\right] I_{[\alpha, \beta]}(t) .
$$

If $h \in N_{c}$ then, in $B_{2}$,

$$
\sqrt{n} \xi_{n}\left(\theta_{0}, P_{h}^{n}\right)=V \circ \tau h+o(1)
$$

using $F\left(n^{-1 / 2} h\right)=F\left(\theta_{0}\right)+n^{-1 / 2} \tau h$. The calculation (9.9) has long been familiar to students of robustness with varying degrees of rigor (cf. Huber (1977) and Serfling (1980)). Hypothesis (9.6) guarantees that identifiability and differentiability hold for $\xi_{n}\left(\theta, P_{h}^{n}\right)$ whenever $h \in N_{c}$. It is now immediate from the basic result of $\S 5$ that the first expression in (9.8) is at least as big as the second. To see why the QMDE is optimal (i.e., to prove the second inequality and hence the theorem), let $h_{n}$ be a sequence in $N_{c}$; we must show that under $P_{h_{n}}^{n}, n^{1 / 2}\left(\hat{\theta}_{n}-\theta_{n h_{n}}\right)$ converges to $T^{-1} \circ \pi \circ V \circ Z$, where $Z$ has the distribution of $W^{0}\left(F\left(\theta_{0} ; t\right)\right)$. Relation (9.9) suggests that if $\xi_{1 n}(\theta)=\hat{F}_{n}^{-1}-F^{-1}(\theta)$, then

$$
n^{1 / 2} \xi_{1 n}\left(\theta_{0}\right)=V\left(n^{1 / 2}\left(\hat{F}_{n}-F\left(\theta_{0}\right)\right)\right)+o(1)
$$


under $P_{h_{n}}^{n}$. Arguments of Shorack can be used to make this rigorous. The process $\xi_{1 n}(\theta)$ therefore satisfies the hypotheses of boundedness (under $P_{h_{n}}^{n}$ ); it satisfies identifiability and differentiability by (9.6). Therefore

$$
n^{1 / 2}\left(\hat{\theta}_{n}-\theta_{0}\right)=-T^{-1} \circ \pi \circ V\left(n^{1 / 2}\left(\hat{F}_{n}-F_{\theta_{0}}\right)\right)+o(1)
$$

by the basic result of $\S 3$. Similarly, if $\xi_{2 n}(\theta)=F^{-1}\left(n^{-1 / 2} h_{n}\right)-F^{-1}(\theta)$, then

$$
n^{1 / 2}\left(\theta_{n h}-\theta_{0}\right)=-T^{-1} \circ \pi \circ V\left(n^{1 / 2}\left[F\left(n^{1 / 2} h_{n}\right)-F\left(\theta_{0}\right)\right]\right)
$$

so together,

$$
n^{1 / 2}\left(\hat{\theta}_{n}-\theta_{0}\right)=-T^{-1} \circ \pi \circ V\left(n^{1 / 2}\left(\hat{F}_{n}-F\left(n^{-1 / 2} h\right)\right)\right)+o(1) .
$$

Since, under $P_{h_{n}}^{n}, n^{1 / 2}\left[\hat{F}_{n}-F\left(n^{-1 / 2} h_{n}\right)\right]$ converges to $W^{0}\left(F\left(\theta_{0}\right)\right)$, the result is proved. Q.E.D.

X. Simple regression. Let $F$ be a fixed known cdf on the line. For each $n$, let $c_{n 1}, \ldots, c_{n n}$ be known real numbers. Let $X_{n 1}, \ldots, X_{n n}$ be real random variables. The simple regression model asserts that, for some $\theta$,

$$
\left\{X_{n i}-c_{n i} \theta, 1 \leq i \leq n\right\} \text { is i.i.d. } F \text {. }
$$

The statistical problem is to "estimate $\theta$ ".

Let $\mu$ be a measure on the line; set $B=L^{2}(\mu)$. Let $d_{n 1}, \ldots, d_{n n}$ be a sequence of numbers such that

$$
\max _{i}\left|d_{n i}\right| \rightarrow 0, \quad \sum d_{n i}^{2}=1
$$

Define the process

$$
\xi_{n}(\theta)(t)=\sum d_{n i}\left[I\left\{X_{n i} \leq t+c_{n i} \theta\right\}-F(t)\right]
$$

the "normalized weighted empirical cdf". $\xi_{n}(\theta)$ is a random element that we suppose belongs to $L^{2}(\mu)$. The minimum distance estimate of $\theta$ is $\hat{\theta}_{n}$, defined by

$$
\inf _{\theta}\left|\xi_{n}(\theta)\right|_{\mu}=\left|\xi_{n}\left(\hat{\theta}_{n}\right)\right|_{\mu} .
$$

Under various hypotheses, Koul-de Wet proved that such an estimate was asymptotically normal. We explain in this section how this asymptotic normality follows from our general structural theorems. In addition, we prove a LAM property which is new.

To state the LAM property let

$$
f \text { be the density of } F
$$

with respect to Lebesgue measure. We suppose $f \in L^{2}(\mu)$. Define a Hilbert space $H=\left\{h \in L^{2}(\mu): \int h f=0\right\}$. Construct probabilities $F(h ; d x)$ by specifying its density $f(h ; x)$ to be

$$
f(h ; x)=f(x)[1+h(x)] .
$$

Define $P_{h}^{n}$, a product measure, so that under $P_{h}^{n}, X_{n i}-c_{n i} \theta_{0}, 1 \leq i \leq n$, are independent with $X_{n i}-c_{n i} \theta_{0}$ distributed as $F\left(d_{n i} h\right)$. Define $(\tau h)(t)=\int^{t} h(s) f(s) d s$, and note that

$$
F\left(d_{n i} h\right)=F+d_{n i} \tau h
$$


Assume $\mu$ finite, for convenience (so $\tau h \in L^{2}(\mu)$ ); define $B$ to be the closure of $\tau H$ in $L^{2}(\mu)$. Then $(\tau, H, B)$ is an abstract Wiener space; if $\left\{Q_{h}\right\}$ is its Gaussian shift experiment, then $Q_{0}$ is the distribution of $W^{0}(F)$. Let $N_{c}=\{h \in H:|h| \leq c\}$. Define $\theta_{n h}$ by

$$
\begin{gathered}
\xi_{n}\left(\theta ; P_{h}^{n}\right)(t)=\sum d_{n i}\left[F\left(d_{n i} h ; t\right)-F\left(t-c_{n i}\left(\theta-\theta_{0}\right)\right)\right], \\
\quad \inf _{\theta}\left|\xi_{n}\left(\theta, P_{h}^{n}\right)\right|_{\mu}=\left|\xi_{n}\left(\theta_{n h}\right)\right|_{\mu} .
\end{gathered}
$$

Let $Z$ be a real Gaussian random variable,

$$
\begin{gathered}
Z \sim N\left(0, \sigma^{2}\right), \\
\sigma^{2}=\left(\frac{1}{|f|_{\mu}}\right)^{4} \iint[F(s \wedge t)-F(s) F(t)] f(s) f(t) \mu(d s) \mu(d t) .
\end{gathered}
$$

The following theorem holds under the conditions given in Koul-de Wet; these conditions, which include restrictions on the $c_{n i}, d_{n i}$, are quite lengthy to state.

(10.11) THEOREM.

$$
\begin{array}{r}
\lim _{c} \liminf _{n} \sup _{\tilde{\theta}_{n}} \int g\left(\delta_{n}\left|\tilde{\theta}_{n}-\theta_{n h}\right|\right) d P_{h}^{n}=E g(|Z|) \\
=\lim _{c} \lim _{n} \sup _{h \in N_{c}} \int g\left(\delta_{n}\left|\hat{\theta}_{n}-\theta_{n h}\right|\right) d P_{h}^{n}
\end{array}
$$

where $\delta_{n}=\Sigma d_{n i} c_{n i}$, and $\hat{\theta}_{n}$ was defined in (10.4).

PROOF. The proof will be sketched only; a number of the calculations can be found in Koul-de Wet. We begin by showing that the first expression in the theorem is bigger than the second. Because of $(10.2)$ and $(10.8), \xi_{n}\left(\theta_{0}, P_{h}^{n}\right)=\tau h$, so condition (5.7) is satisfied as well as the condition of boundedness (2.4). Moreover, under mild conditions,

$$
\begin{aligned}
\xi_{n}\left(\theta, P_{h}^{n}\right)-\xi_{n}\left(\theta_{0}, P_{h}^{n}\right) & =-\sum d_{n i}\left[F\left(t-c_{n i}\left(\theta-\theta_{0}\right)\right)-F(t)\right] \\
& \doteq \sum c_{n i} d_{n i}\left(\theta-\theta_{0}\right) f(t) \\
& =\delta_{n}\left(\theta-\theta_{0}\right) f(t) \quad \text { in } L^{2}(\mu),
\end{aligned}
$$

so $\xi_{n}$ is differentiable with derivative $\eta=f$ as defined in (3.2). The hypothesis of identifiability is more delicate and depends on relations between $c_{n i}, d_{n i}$, as well as some smoothness in $F$. It can be checked under the conditions of Koulde Wet. Therefore, the processes $\xi_{n}\left(\theta, P_{h}^{n}\right)$ satisfy the condition of $\S 5$, so that the first expression in the statement of the theorem is at least as big as

$$
\int g\left(\left|T^{-1} \circ \pi x\right|\right) Q_{0}(d x)
$$

where $\pi$ is projection to span $\eta$ in $L^{2}(\mu)$, and $T$ is the usual operator (2.10). But here,

$$
T^{-1} \circ \pi x=\langle x, f\rangle_{\mu} /|f|_{\mu}^{2}, \quad x \in L^{2}(\mu),
$$

by (2.12), and $Q_{0}$ is the distribution of $W^{0} \circ F$. Therefore, (10.12) is equal to $E g(|Z|)$, where $Z=\left\langle W_{F}, f\right\rangle_{\mu} /|f|_{\mu}^{2}$. By an elementary calculation using the form of 
the covariance function of $W_{F}$ and the form of the $L^{2}(\mu)$ inner product, it is easy to see that the $Z$ just given has the distribution (10.10). This establishes the LAM lower bound.

To show that $\hat{\theta}_{n}$, defined by (10.4), is LAM, let $h_{n}$ be a sequence in $N_{c}$. It is necessary to show only that $\delta_{n}\left(\hat{\theta}_{n}-\theta_{n h_{n}}\right)$ converges to $Z$ under $P_{h_{n}}^{n}$. This was shown by Koul-de Wet for $\delta_{n}\left(\hat{\theta}_{n}-\theta_{n}\right)$ under $P_{0}^{n}$; we content ourselves here with showing how to apply the abstract approach of $\S 2$.

Under $P_{h_{n}}^{n}$, one first shows that the process $\xi_{n}(\theta)$, defined in (10.3), satisfies the conditions of boundedness, identifiability, and differentiability, with derivative $\delta_{n} f$ (same as for $\xi_{n}\left(\theta, P_{h}^{n}\right)$ introduced in (10.9)). This undertaking involves a fair amount of work. To see that differentiability is at least plausible, write

$$
\xi_{n}\left(\theta_{0}+\theta\right)-\xi_{n}\left(\theta_{0}\right)=\sum d_{n i} I\left\{c_{n i} \theta_{0}+t \leq X_{n i} \leq c_{n i}\left(\theta+\theta_{0}\right)+t\right\},
$$

which is possible if $c_{n i} \theta \geq 0$; it is then plausible that this last expression is approximable, in probability, in $L^{2}(\mu)$, by $\Sigma c_{n i} d_{n i} f(t) \theta$. The hypothesis of boundedness, under $P_{h_{n}}^{n}$ can be checked, e.g., by using the CLT in Hilbert space to show that, under $P_{h_{n}}^{n_{n}}$,

$$
\xi_{n}^{*}(\theta) \equiv \sum d_{n i}\left[I\left\{X_{n i} \leq t+c_{n i} \theta_{0}\right\}-F\left(n^{-1 / 2} h_{n}\right)\right]
$$

converges in $L^{2}(\mu)$ to $W^{0}(F)$; the treatment is a minor variant of the usual arguments involving the empirical cdf. Identifiability is more involved; see Koul-de Wet for applicable arguments. Assuming this done, the theory of $\S 2$ shows

$$
\delta_{n}\left(\hat{\theta}_{n}-\theta_{0}\right)=-T^{-1} \circ \pi \circ \xi_{n}\left(\theta_{0}\right)+o(1)
$$

As in the first part of the proof the processes $\xi_{n}\left(\theta, P_{h_{n}}^{n}\right)$ satisfy the basic three hypotheses, so

$$
\delta_{n}\left(\theta_{n h}-\theta_{0}\right)=-T^{-1} \circ \pi \circ \xi_{n}\left(\theta_{0}, P_{h_{n}}^{n}\right)+o(1)
$$

Therefore

$$
\delta_{n}\left(\hat{\theta}_{n}-\theta_{n h}\right)=-T^{-1} \circ \pi \circ \xi_{n}^{*}(\theta) \Rightarrow-T^{-1} \circ \pi \circ W_{F},
$$

whence the result. Q.E.D.

(10.13) REMARK. It may be worthwhile at this point to make more explicit the sense in which the present "estimate of $\theta$ " is optimal. The model asserts that the data $X_{n 1}, \ldots, X_{n m}$ are independent, with distributions given by the vector $V_{n}(\theta)=F\left(t-c_{n i} \theta\right), \ldots, F\left(t-c_{n n} \theta\right)$ for some $\theta$. On the other hand, due to "data contamination", it may happen that the data are independent, with distributions governed by a sequence of cdf's $G_{n}=\left(G_{n 1}, \ldots, G_{n n}\right)$. In these circumstances one announces the statistical goal to be that of finding the point $\theta$ such that the vector of cdf's, $V_{n}(\theta)$, "best fits the data", i.e., such that $V_{n}(\theta)$ is as "close as possible" to $G_{n}$. Many notions of distance could be chosen here; the distance used in this section was the $L^{2}(\mu)$ distance between $\Sigma d_{n i} G_{n i}$ and $\Sigma d_{n i} F\left(\cdot-c_{n i} \theta\right)$. When the $d_{n i}$ have been chosen, the main result says that the estimate $\hat{\theta}_{n}$ of $(10.4)$ does the best job under the situation where there is rather severe local contamination (i.e., quite a few $G_{n}$ are allowed), and when the goal is the one of "best fit" just mentioned. It seems quite an irrelevant undertaking, in the present context, to compare the asymptotic variances of the present estimate with others that may be invented. 
One could choose the $d_{n i}$ (subject to $(10.2)$ ) so that $\delta_{n}$ is as large as possible; this would ensure that the estimate $\hat{\theta}_{n}$ has the "fastest convergence" to $\theta_{n h}$. This choice is $d_{n i}=c_{n i} /\left(\Sigma c_{n i}^{2}\right)^{1 / 2}$ by the Schwarz inequality. On the other hand, the selection of $d_{n i}$ determines what you mean by "best fit" described in the preceding paragraph; and this newly proposed choice of $d_{n i}$ may or may not embody a reasonable notion of "best fit". It is not unthinkable that practitioners would prefer $d_{n i}=n^{-1 / 2}$, $1 \leq i \leq n$, since this yields a readily grasped notion of closeness. In other words, the choice of $d_{n i}$ should depend on what you think the best notion of distance is for fitting the model to the data. If you, for example, choose the $d_{n i}$ unequal, this means you want certain of the $G_{i}$ to be better "fitted" by the model than others; there may be good reason for this in some cases, but such a reason would not depend on the size of $\delta_{n}$.

To put it another way: when you change the $d_{n i}$, you change the notion of which $\theta$ you are trying to estimate. You should decide at the beginning, on the basis of your statistical goals, which $\theta$ you want to estimate. For this it is irrelevant that, if someone suggests estimating a different $\theta$, then some estimator for that $\theta$ has, e.g., a faster convergence rate.

XI. Estimates based on spectral functions. Let $X_{1}, \ldots, X_{n}$ be a real stationary Gaussian sequence with mean 0 . Assume that the spectral function is of the form $F(\theta ; t),-\pi \leq t \leq \pi$, for some $\theta \in \Theta$ an open subset of $R^{d}$; then

$$
E_{\theta} X_{k} X_{k+j}=\int_{-\pi}^{\pi} e^{i j t} F(\theta ; d t) .
$$

Assume $F(\theta ; 0)=0$; this reduction involves only a renormalization. Define, for any spectral function $G(G(o)=0)$, the minimum distance functional $\theta(G)$ by

$$
\inf _{\theta}|G-F(\theta)|_{\mu}=|G-F(\theta(G))|_{\mu},
$$

where $\mu$ is a finite measure on $[0, \pi]$ and $\|_{\mu}$ is the norm of $L^{2}(\mu)$. Define

$$
\hat{F}_{n}(t)=(2 \pi n)^{-1} \int_{0}^{t}\left|\sum_{k=1}^{n} X_{k} e^{-i y k}\right|^{2} d y
$$

Then $\hat{F}_{n}$ is a random element of $L^{2}(\mu)$ and is a well-known spectral function estimate (closely analogous to the empirical cdf as an estimate of a cdf, discussed in $\S 6)$. Define $\hat{\theta}_{n}$, estimate of $\theta$, by

$$
\hat{\theta}_{n}=\theta\left(\hat{F}_{n}\right)
$$

The main result of this section is that the estimator $\hat{\theta}_{n}$ is asymptotically normal and LAM; these results appear to be new. The development is based on the general theory of $\S \S 3,5$ and illustrates the fact that "independent observations" have nothing to do with the basic framework.

To state these results, fix $\theta_{0}$. Assume that

(11.5) (a) $F\left(\theta_{0} ; \cdot\right)$ has density $f\left(\theta_{0} ; t\right)$ on $[0, \pi]$ with respect to Lebesgue measure, and $\int_{0}^{\pi} f^{2}(t) d t<\infty$.

(b) If $F\left(\theta_{n}\right) \rightarrow F\left(\theta_{0}\right)$ in $L^{2}(\mu)$, then $\theta_{n} \rightarrow \theta_{0}$.

(c) There exists $\eta=\left(\eta_{1}, \ldots, \eta_{d}\right), \eta_{i}$ linearly independent elements of $L^{2}(\mu)$ such that $F(\theta)-F\left(\theta_{0}\right)=\langle\theta, \eta\rangle+o\left(\left|\theta-\theta_{0}\right|\right)$. 
Let $H$ be the Hilbert space of all real functions $h$ on $[0, \pi]$ such that $\int_{0}^{\pi} h^{2} f^{2}<\infty$. For $h \in H$ define spectral functions $F(h ; t)$ by

$$
F(h ; d t)=[1+h(t) f(t)] f(t) .
$$

Let

(11.7) $P_{h}^{n}=$ distribution of the stationary Gaussian sequence $X_{1}, \ldots, X_{n}$ when the spectral function is $F\left(h n^{-1 / 2} ; \cdot\right)$.

Next, define $\tau$, a linear map of $H$ to $L^{2}(\mu)$, by

$$
(\tau h)(t)=\int_{0}^{t} h f^{2}
$$

so that

$$
F(h)=F\left(\theta_{0}\right)+\tau h .
$$

Let $B$ be the closure of $\tau(H)$ in $L^{2}(\mu)$. Then $(\tau, H, B)$ is an abstract Wiener space. Let $\left\{Q_{h}, h \in H\right\}$ be its standard Gaussian shift experiment: we may characterize $Q_{0}$ as follows. Let $W$ be standard Brownian motion on the line. Set

$$
M(t)=2 \pi \int_{0}^{t} f^{2}(s) d s, \quad W_{F}(t)=W(M(t)), \quad 0 \leq t \leq \pi .
$$

Then $Q_{0}$ is the distribution of $W_{F}$, a fact easy to check.

Finally, fix $\alpha, 1 / 2<\alpha<1$; let $N_{c}=\left\{h \in H:|h(s)-h(t)| \leq c|s-t|^{\alpha},|h|_{\mu} \leq c\right\}$. Then $\bigcup_{c} N_{c}$ is a dense subspace of $H$. Define

$$
\theta_{n h}=\theta\left(F\left(n^{-1 / 2} h\right)\right) \text {. }
$$

(11.12) THEOREM. Under the hypotheses above,

$$
\begin{gathered}
\lim _{c} \liminf _{n} \sup _{\tilde{\theta}_{n}} \int g\left(n^{1 / 2}\left|\tilde{\theta}_{n}-\theta_{n h}\right|\right) d P_{h}^{n}=E g\left(\left|T^{-1} \circ \pi \circ W_{F}\right|\right) \\
=\lim _{c} \lim _{n} \sup _{h \in N_{c}} \int g\left(n^{1 / 2}\left|\hat{\theta}_{n}-\theta_{n h}\right|\right) d P_{h}^{n}
\end{gathered}
$$

where, as usual, the inf in the first expression is over all estimates of $\left\{\theta_{n h}\right\}$ and $g$ is an (bounded) increasing function on the line; $\pi$ as usual is the projection to span $\eta_{i}$, and $T$ is defined by $(2.10)$.

(11.13) REMARK. As in the other situations of this paper, the statistical goal here is one of "best fit". A simple model asserts that $X_{1}, \ldots, X_{n}$ is stationary Gaussian with spectral function $F(\theta)$. However, it is presumed that the model does not actually fit the data precisely - the data is stationary Gaussian (this hypothesis can be weakened, but is outside the scope of this paper) but the actual spectral function is alleged to differ some from $\left\{F_{\theta}, \theta \in \Theta\right\}$. Because the model $\left\{F_{\theta}\right\}$ has appealing simplicity, or for other reasons, one still desires to fit it to the data as best one can. Here this goal is formulated technically by (11.2): one wants the spectral function of the form $\left\{F_{\theta}, \theta \in \Theta\right\}$ that comes 'closest' to the actual spectral function. It is in this context that the optimality result is to be understood.

PROOF. We begin by showing that the first expression in (11.12) exceeds the second. Define

$$
\xi_{n}\left(\theta, P_{h}^{n}\right)=F\left(n^{-1 / 2} h\right)-F(\theta) .
$$


Because of (11.9), this process satisfies (5.19) with $V$ the identity. The hypotheses of identifiability and differentiability in $\S 3$ (with derivative $\eta$ ) follow from (11.5); the hypothesis of boundedness is trivial. Moreover, results of Davies (1973) show, via Lemma (4.1), that the experiments $\left\{P_{h}^{n}, h \in H_{0}\right\}$ converge to $\left\{P_{h}, h \in H_{0}\right\}$, where $H_{0}=\bigcup_{c>0} N_{c}$. The desired inequality is now immediate from (5.20).

Next, let $h_{n}$ be a sequence in $N_{c}$; to finish the proof of the theorem it is necessary (cf. (5.18)ff) only to show that, under $P_{h_{n}}^{n}$,

$$
n^{1 / 2}\left(\hat{\theta}_{n}-\theta_{n h_{n}}\right) \Rightarrow T^{-1} \circ \pi \circ W_{F} .
$$

Let $\xi_{1 n}(\theta)=\hat{F}_{n}-F(\theta)$. Arguments of Ibragimov (1963) show that, under $P_{h_{n}}^{n}$,

$$
\left(\hat{F}_{n}-F\left(n^{-1 / 2} h_{n}\right)\right) n^{1 / 2} \Rightarrow W_{F} \quad \text { in } L^{2}(\mu) \text {. }
$$

This easily implies the hypotheses of boundedness for $\xi_{1 n}(\theta)$. Since the hypotheses of differentiability and identifiability follow as in the first part of the proof, we have

$$
\left(\hat{\theta}_{n}-\theta_{0}\right)=-T \circ \pi \circ \xi_{1 n}\left(\theta_{0}\right)+o\left(n^{-1 / 2}\right)
$$

by the main result of $\S 5$. Similarly, define $\xi_{2 n}(\theta)=F\left(n^{-1 / 2} h_{n}\right)-F(\theta)$; this satisfies the three basic hypotheses too, so

$$
\left(\theta_{n h_{n}}-\theta_{0}\right)=-T \circ \pi \circ \xi_{2 n}\left(\theta_{0}\right)+o\left(n^{-1 / 2}\right) .
$$

Then (11.14) is immediate from (11.15)-(11.17). Q.E.D.

XII. Hellinger metric. Let $\left\{P_{\theta}, \theta \in \Theta\right\}$ be a family of probabilities, on some Euclidean space $S$, indexed by $\Theta$, an open subset of $R^{d}$. Assume there is a sigmafinite measure $\mu$ such that each $P_{\theta}$ is absolutely continuous with respect to $\mu$ with density $f_{\theta}=f(\theta)$ :

$$
f(\theta ;)=d P_{\theta} / d \mu .
$$

Let $\theta_{0}$ be a subset of $\theta$ of the form

$$
\Theta_{0}=\Theta \cap M,
$$

where $M$ is a subspace of $R^{d}$ having dimension $s<d$. This section treats the problem of trying to fit the model $\left\{P_{\theta}, \theta \in \Theta\right\}$ as best as one can using the "simpler" model $\left\{P_{\theta}, \theta \in \Theta_{0}\right\}$. Such a problem arises in certain areas of applied statistics (e.g., multivariate analysis) where the data is probably governed by a parametric model $\left\{P_{\theta}, \theta \in \theta\right\}$ where the dimension of $\theta$ is so large as to be unhelpful in understanding the basic shape of the data; one therefore desires to use a rougher model-with parameter set $\boldsymbol{\theta}_{0}$ having much lower dimension-as a workable approximation.

The metric to be used for this study is the Hellinger metric, which is the basic one for much of classical parametric estimation (cf. Le Cam (1973)). The Hellinger distance between $P_{\theta}$ and $P_{\theta^{\prime}}$ is the $L^{2}(\mu)$ distance between $f_{\theta}^{1 / 2}, f_{\theta^{\prime}}^{1 / 2}$. The relevant minimum distance functional is then $\varsigma\left(\theta^{\prime}\right)$ defined by

$$
\inf _{\theta \in \Theta_{0}}\left|f^{1 / 2}\left(\theta^{\prime}\right)-f^{1 / 2}(\theta)\right|_{\mu}=\left|f^{1 / 2}\left(\theta^{\prime}\right)-f^{1 / 2}\left(\varsigma\left(\theta^{\prime}\right)\right)\right|_{\mu},
$$

i.e., we naturally want to find $\varsigma\left(\theta^{\prime}\right)$, the point in $\theta_{0}$ so that $P\left(\varsigma\left(\theta^{\prime}\right)\right)$ is closest to $P\left(\theta^{\prime}\right)$ in the Hellinger metric. 
We shall treat the estimation of $\varsigma(\theta)$ under the following hypotheses. Assume for each point $\theta_{1} \in \Theta$ :

(12.4) (a) If $f^{1 / 2}\left(\theta_{n}\right)$ converges to $f^{1 / 2}\left(\theta_{1}\right)$ in $L^{2}(\mu)$, then $\theta_{n} \rightarrow \theta_{1}$.

(b) There exists $\eta\left(\theta_{1}\right)=\left(\eta_{1}\left(\theta_{1}\right), \ldots, \eta_{d}\left(\theta_{1}\right)\right), \eta_{i}\left(\theta_{1}\right) \in L^{2} \mu$, linearly independent, such that

Fix now $\theta_{0} \in \Theta$.

$$
f^{1 / 2}(\theta)-f^{1 / 2}\left(\theta_{1}\right)=\left\langle\theta-\theta_{1}, \eta\left(\theta_{1}\right)\right\rangle+o\left(\left|\theta-\theta_{1}\right|\right)
$$

(12.5) Let $\Gamma=$ matrix with entries $\left\langle\eta_{i}, \eta_{j}\right\rangle_{\mu}, \eta=\eta\left(\theta_{0}\right)=\left(\eta_{1}, \ldots, \eta_{d}\right)$. Because $\eta_{i}$ are linearly independent, $\Gamma$ is nonsingular. For convenience we assume

$$
\Theta_{0}=\Theta \cap M \quad \text { with } M=\left\{y \in R^{d}: y=\left(y_{1}, \ldots, y_{s}, o, o, \ldots, o\right)\right\} \text {. }
$$

This assumption can always be satisfied by means of an appropriate reparametrization (which can be chosen independently of $\theta_{0}$ ).

Let $H$ be the Hilbert space consisting of $R^{d}$ with the usual Euclidean metric. Let $\Sigma$ be the matrix

$$
\Sigma=\frac{1}{4} \Gamma^{-1}
$$

Let $B$ be $R^{d}$ with norm

$$
|x|_{B}=\left|\Sigma^{1 / 2} x\right|_{0},
$$

where ||$_{0}$ is Euclidean norm. Define $\tau: H \rightarrow B$ by $\tau h=h$. Then $(\tau, H, B)$ is an abstract Wiener space; if $\left\{Q_{h}, h \in H\right\}$ is its Gaussian shift experiment then

$$
Q_{0} \text { is } N(0, \Sigma) \text { on } R^{d} \text {. }
$$

For $h \in H$ define

$$
P_{h}^{n}(d x)=\prod_{i}^{n} f\left(\theta_{0}+n^{-1 / 2} h, x_{i}\right) d x_{i}
$$

Define matrices

$$
\begin{aligned}
\Gamma_{s} & =\left\{\left\langle\eta_{i}, \eta_{j}\right\rangle: 1 \leq i \leq s, 1 \leq j \leq s\right\}, \\
\Gamma_{s d} & =\left\{\left\langle\eta_{i}, \eta_{j}\right\rangle: 1 \leq i \leq s, 1 \leq j \leq d\right\}
\end{aligned}
$$

so $\Gamma_{s d}$ maps $R^{d}$ to $R^{s}$. Then, for any point $x \in R^{d}$, one may calculate

$$
A x \equiv \Gamma_{s}^{-1} \circ\left(\Gamma_{s d} x\right) \text {. }
$$

Define $N_{c}=\{h \in H:|h| \leq c\}$, and let $\hat{\theta}_{n}$ be the classical 1-step maximum likelihood estimator; this well-known estimator is a bit complicated to describe - see Le Cam (1969).

(12.13) THEOREM.

$$
\begin{gathered}
\lim _{c \uparrow \infty} \liminf _{n} \sup _{\tilde{\theta}_{n}} \int g\left(n^{1 / 2}\left|\tilde{\theta}_{n}-\theta_{n h}\right|\right) d P_{h}^{n}=\int g(|A x|) Q_{0}(d x) \\
=\lim _{c \uparrow \infty} \lim _{n} \sup _{h \in N_{c}} \int g\left(n^{1 / 2}\left|\varsigma\left(\hat{\theta}_{n}\right)-\theta_{n h}\right|\right) d P_{h}^{n},
\end{gathered}
$$

where $\theta_{n h}=\varsigma\left(\theta_{0}+h n^{-1 / 2}\right)$, and $\varsigma$ was defined in (12.3).

Evidently, the result asserts that $\varsigma\left(\hat{\theta}_{n}\right)$ is $L A M$. 
PROOF. We begin by showing that the first expression in the theorem is at least as big as the second. For this, note first that the experiments $\left\{P_{h}^{n}, h \in H\right\}$ converge to $\left\{Q_{0}, h \in H\right\}$. Define

$$
\xi_{n}\left(\theta, P_{h}^{n}\right)=f^{1 / 2}\left(n^{-1 / 2} h\right)-f^{1 / 2}(\theta), \quad \theta \in \Theta_{0} .
$$

Because of (12.4)(b),

$$
n^{1 / 2} \xi_{n}\left(\theta_{0}, P_{h}^{n}\right)=\langle h, \eta\rangle+o(1)=V \circ \tau h,
$$

where $\tau h=h, V x=\langle x, \eta\rangle$. Therefore $\xi_{n}$ satisfies (5.19). It is also clear from (12.4) that $\xi_{n}$ satisfies identifiability, boundedness, and differentiability with derivative $\eta_{0}=\left(\eta_{1}, \ldots, \eta_{s}, o, o, \ldots, o\right)$ (this derivative is taken in $\theta_{0}$, not $\theta$ ). Let $\pi=$ projection in $L^{2}(\mu)$ to span $\eta_{1}, \ldots, \eta_{s}$, and let $T$ be as in (2.10). Then the basic result of $\S 5$ asserts that the first expression in (12.13) is bounded below by $\int g\left(\left|T^{-1} \circ \pi \circ V x\right|\right) P_{0}(d x)$. Using the evaluation of $T^{-1} \circ \pi$ given in (2.19), and the explicit form of $V$ above, one obtains from this the expression given in the theorem.

To finish the proof it is now necessary to show only that if $h_{n}$ is a sequence in $N_{c}$, then $n^{1 / 2}\left[\varsigma\left(\hat{\theta}_{n}\right)-\varsigma\left(\theta_{n h_{n}}\right)\right]$ converges under $P_{h_{n}}^{n}$, to $T^{-1} \circ \pi \circ V \circ Z$, where $Z$ has distribution $P_{0}$ (see $(5.18)$ ff). To do this, use the well-known fact that

$$
n^{1 / 2}\left[\hat{\theta}_{n}-\left(\theta_{0}+h_{n} n^{-1 / 2}\right)\right] \Rightarrow Z
$$

see Le Cam (1969) or Roussas (1972).

Next, set $\xi_{1 n}(\theta)=f^{1 / 2}\left(\hat{\theta}_{n}\right)-f^{1 / 2}(\theta)$; this satisfies, under $P_{h_{n}}^{n}$, the hypotheses of differentiability (with derivative $\eta_{0}$ ), identifiability, and boundedness (because of (12.14) and the fact that $h_{n}$ is bounded). Therefore, using (12.4)(b) and (12.14),

$$
\begin{aligned}
n^{1 / 2}\left[\varsigma\left(\hat{\theta}_{n}\right)-\theta_{0}\right] & =-T^{-1} \circ \pi \circ \xi_{1 n}\left(\theta_{0}\right)+o(1) \\
& =-T^{-1} \circ \pi \circ V\left(\hat{\theta}_{n}-\theta_{0}\right)+o(1) .
\end{aligned}
$$

On the other hand, if $\xi_{2 n}(\theta)=f^{1 / 2}\left(\theta_{0}+h_{n} n^{-1 / 2}\right)-f^{1 / 2}(\theta)$ the three hypotheses are again satisfied, so

$$
n^{1 / 2}\left(\theta_{n h_{n}}-\theta_{0}\right)=-T^{-1} \circ \pi \circ V\left(n^{-1 / 2} h n\right) .
$$

The desired result is now immediate from (12.14)-(12.16).

(12.17) REMARK. It is of interest to consider a somewhat different estimator: to estimate $\theta_{n h} \in \theta_{0}$, first calculate $\hat{\theta}_{n}$, the one step MLE for the big model $\left\{P_{\theta}, \theta \in \theta\right\}$; then estimate $\theta_{n h}$ by the first $s$ coordinates of $\hat{\theta}_{n}$. If $\eta_{s+1}, \ldots, \eta_{d}$ are orthogonal to $\eta_{1}, \ldots, \eta_{s}$, then facts given in the foregoing proof easily show that such an estimator is also LAM. On the other hand, if the aforementioned orthogonality fails, the newly proposed estimator and $\zeta\left(\hat{\theta}_{n}\right)$ are not asymptotically equivalent (and the new one will not be LAM in the present framework).

It is possible, by a reparametrization, to make $\eta_{s+1}, \ldots, \eta_{d}$ orthogonal to $\eta_{1}, \ldots$, $\eta_{s}$. This reparametrization will change the definition of what is being estimated, and, moreover, the reparametrization will depend on $\theta_{0}$. Evidently, the statistical meaning of a local asymptotic investigation is completely destroyed if the definition of what is being estimated is changed for the convenience of the point $\theta_{0}$ chosen for the local analysis. 
XIII. Further methods in the independent case. Let $\theta$ be an open subset of $R^{d}$ and $\left\{P_{\theta}, \theta \in \Theta\right\}$ a family of probabilities on $R^{s}$. Let $X_{1}, \ldots, X_{n}$ be independent random $R^{s}$-valued random variables with common distribution $G$. As in other sections, the goal is to 'fit' the model $\left\{P_{\theta}\right\}$ as best one can to the actual data distribution. In $\S 6$ this was done by matching up cdf's as close as one could; in $\S 9$ we "fitted" the model to the data by matching the quantiles. Many other methods are possible and have practical importance. This section exhibits some of the possibilities together with natural LAM estimators.

We begin by giving the relevant abstract structure; many illustrations of it follow.

Let $(Y, y)$ be a measure space, $\mu$ a measure on $y$. $Y$ will be assumed Euclidean, but this is not really necessary. Let $\zeta$ be a mapping defined on probabilities on $R^{s}$ such that if $G$ is a probability, then $\varsigma(G)$ is a real measurable function on $Y$ : its value at $t \in Y$ is $\zeta(G)(t)$. $\varsigma$ need not be defined on all probabilities-only on the probabilities $P_{\theta}$ and a certain few others to be described below. We assume that $\varsigma(G)$ satisfies

$$
\int[\zeta(G)(t)]^{2} \mu(d t)<\infty
$$

so $\zeta$ is an $L^{2}(\mu)$-valued functional defined on certain measures. Define the functional $\theta(G)$ by

$$
\inf _{\theta}\left|\zeta(G)-\varsigma\left(P_{\theta}\right)\right|_{\mu}=\left|\zeta(G)-\varsigma\left(P_{\theta(G)}\right)\right|_{\mu},
$$

where, of course, it is assumed that $\zeta$ is defined on $G$. The statistical problem is to estimate $\theta(G)$.

The basic hypothesis on $\zeta(G)$ is that it is essentially given locally by a recipe of the type $\varsigma(G)(t)=\int \psi(t, x) G(d x)$ for some measurable function $\psi$ on $Y \times R^{s}$. This will be described next, but it takes a bit of time. The classic $M, L$ and $R$ functionals have this simple structure, but the class to be described is much broader, including even the functionals of $\S \S 6,9$.

The probabilities $P_{\theta}$, which are defined on the Borel sets of $R^{s}$, are assumed absolutely continuous with respect to a sigma-finite (nonatomic) measure $\nu$. As in previous LAM developments, fix $\theta_{0}$. Let

$$
f\left(\theta_{0}, x\right)=\text { density of } P_{\theta_{0}} .
$$

Let

$$
\begin{aligned}
& \psi(t, x) \text { be a real (or complex) jointly } \\
& \text { measurable function on } Y \times R^{s} \text {. }
\end{aligned}
$$

This function depends on $\theta_{0}$ also. Assume that, for each $t \in y$,

(13.5) (a) $\int \psi^{2}(t, x) d P_{\theta_{0}}(x)<\infty$,

(b) $\int \psi(t, x) f\left(\theta_{0}, x\right) \nu(d x)=0$.

Define $H$ to be the closure (in $\left.L^{2}\left(P_{\theta_{0}}\right)\right)$ of span $\{\psi(t, \cdot): t \in Y\}$. Note that any $\psi$ that satisfies $(13.5)$ (a) can be made to satisfy $(13.5)(\mathrm{b})$ merely by subtracting from it the function of $t$ given in (b). Note also that $H$ here is a sub-Hilbert space of the one given in $\S 6$. Define $\tau: H \rightarrow L^{2}(Y, \mu)$ by

$$
(\tau h)(t)=\langle\psi(t, \cdot), h\rangle_{H} .
$$


Note that $\tau$ is one-to-one on $H$, since if $\langle\psi(t ; \cdot), h\rangle_{H}=0$ for all $t$, then $h \in H$ is orthogonal to $H$. Define

$$
B=\text { closure of } \tau H \text { in } L^{2}(\mu) .
$$

Define the mapping $K(s, t), s, t \in Y$, by

$$
K(s, t)=\langle\psi(t ; \cdot), \psi(s ; \cdot)\rangle_{H} .
$$

Then, since $\tau$ is a Hilbert-Schimdt operator, $(\tau, H, B)$ is an abstract Wiener space; if $\left\{Q_{h}, h \in H\right\}$ is its Gaussian shift experiment, then $Q_{0}$ is a Gaussian measure on $L^{2}(Y, \mu)$ with covariance operator $S$ given by

$$
(S g)(t)=\int K(s, t) g(s) \mu(d s) \text {. }
$$

Alternately, we can characterize $Q_{0}$ as follows. Let $X_{1}, \ldots, X_{n}$ be i.i.d. random variables with distribution $P_{\theta_{0}}$; then $\left\{\psi\left(\cdot ; X_{i}\right)\right\}$ is an i.i.d. sequence of $L^{2}(\mu)$-valued random variables. A routine application of the central limit theorem for Hilbert space (cf., Parthasarathy (1967)), shows that

(13.10) $n^{-1 / 2} \Sigma \psi\left(\cdot ; X_{i}\right)$ converges in distribution (on $\left.L^{2}(\mu)\right)$ to a random variable having distribution $Q_{0}$.

For $h \in H$ define probabilities $P_{h}$ by specifying that the density of $P_{h}$ is $f(h ; \cdot)$ given by

$$
f(h ; x)=f\left(\theta_{0} ; x\right)[1+h(x)]
$$

Let

$$
P_{h}^{n}=n \text {-fold product measure of } P_{n^{-1 / 2} h} \text {. }
$$

Then $\left\{P_{h}^{n}\right\}$ converges to $\left\{Q_{h}\right\}$. Let $H_{0}$ be a dense subspace of $H$.

We can now introduce our hypotheses on the functional $\varsigma$ and the family $\left\{P_{\theta}\right\}$. Assume first, for each $h \in H_{0}$,

$$
\begin{aligned}
\lim _{n \rightarrow \infty}[\zeta & \left.\left(P_{n^{-1 / 2} h}\right)(t)-\varsigma\left(P_{\theta_{0}}\right)(t)\right] n^{1 / 2} \\
& =\int \psi(t ; x) h(x) f_{\theta_{0}}(x) \nu(d x) \equiv(\tau h)(t),
\end{aligned}
$$

convergence in $L^{2}(Y, d \mu)$.

This hypothesis is much weaker than the hypothesis that the functional $G \rightarrow$ $\varsigma(G)$ be Gateaux differentiable at $G$. In most applications $\varsigma$ is not even defined on all $G$; we need only that $\varsigma$ be defined on $\left\{P_{\theta}\right\}$ and on the measures $P_{n^{-1 / 2} h}$, $h \in H_{0}$-a considerably smaller class.

Assume further: there exists $\eta=\left(\eta_{1}, \ldots, \eta_{d}\right), \eta_{i} \in L^{2}(Y, \mu)$, such that

$$
\varsigma\left(P_{\theta}\right)-\varsigma\left(P_{\theta_{0}}\right)=\left\langle\theta-\theta_{0}, \eta\right\rangle+o(|\theta-\theta|) \text {. }
$$

Often there is a vector $h_{0}=\left(h_{1}, \ldots, h_{d}\right)$ such that, for $\theta$ close to $\theta_{0}, f_{\theta} \doteq$ $f_{\theta_{0}}\left[1+\left\langle\theta-\theta_{0}, h_{0}\right\rangle\right]$; this is true, for example, when $\left\{P_{\theta}\right\}$ is quadratic mean differentiable. When such an approximation holds, a variant of (13.13) often holds as well (even though $\left\langle\theta-\theta_{0}, h_{0}\right\rangle$ will usually not belong to $H_{0}$ ), and in this case $\left\langle\theta-\theta_{0}, \eta(t)\right\rangle=\int \psi(t ; x)\left\langle\theta-\theta_{0}, h_{0}\right\rangle f_{\theta_{0}}(x) \nu(d x)$, an explicit form for $\eta$. Finally, assume:

(13.15) If $\varsigma\left(P_{\theta_{n}}\right)$ converges to $\varsigma\left(P_{\theta_{0}}\right)$ in $L^{2}(Y, \mu)$, then $\theta_{n} \rightarrow \theta_{0}$.

Let $N_{c}, c>0$, be defined by $N_{c}=\left\{h \in H_{0}:|h| \leq c\right\}$. Let $\pi$ be the projection in $L^{2}(\mu)$ to span $\eta_{i}$, and define $T$ as in (2.10). 
(13.16) THEOREM (LAM LOWER BOUND). For any increasing function $g$, $\lim _{c} \liminf _{n} \sup _{\tilde{\theta}_{n}} \int g\left(n^{1 / 2}\left|\tilde{\theta}_{n}-\theta_{n h}\right|\right) d P_{h}^{n} \geq \int g\left(\left|T^{-1} \circ \pi x\right|\right) Q_{0}(d x)$, where $\theta_{n h}=\theta\left(P_{n^{-1 / 2} h}\right)$.

(13.17) REMARK. The neighborhoods $\left\{P_{h}^{n}: h \in N_{c}\right\}$ can be replaced by a bigger collection of product measures $\left\{G^{n}\right\}$ and the theorem remains true (of course $d P_{h}^{n}$ is replaced by $d G^{n}, \theta_{n h}$ by $\theta(G)$, and so forth).

PROOF. If one defines $\xi\left(P_{h}^{n}, \theta\right)=\varsigma\left(P_{n^{-1 / 2} h}\right)-\varsigma\left(P_{0}\right)$, then

$$
n^{1 / 2} \xi_{n}\left(P_{h}^{n}, \theta_{0}\right)=n^{1 / 2} \xi_{n}\left(P_{0}^{n}, \theta_{0}\right)+\tau h+o(1)
$$

so (5.19) holds. The hypotheses of boundedness, identifiability, and differentiability hold because of (13.13)-(13.15), so the result is immediate from the development of $\S 5$.

Let $\hat{F}_{n}(d x)$ be the empirical measure of $X_{1}, \ldots, X_{n}$ :

$$
\hat{F}_{n}(A)=n^{-1} \Sigma I_{A}\left(X_{i}\right), \quad A \text { a Borel set of } R^{s} .
$$

Make the assumption:

(13.19) If $h_{n}$ is a sequence in $N_{c}$, then

$$
\begin{gathered}
n^{1 / 2}\left[\zeta\left(\hat{F}_{n}\right)-\varsigma\left(P_{n^{-1 / 2} h_{n}}\right)\right] \Rightarrow Q_{0} \quad \text { under } P_{h_{n}}^{n}, \\
n^{1 / 2}\left[\varsigma\left(P_{n^{1 / 2} h_{n}}\right)-\varsigma\left(P_{\theta_{0}}\right)\right]=\tau h_{n}+o(1) .
\end{gathered}
$$

Except for regularity problems, one would expect such a hypothesis to hold. Indeed, one needs 'only' that $\varsigma\left(\hat{F}_{n}\right)-\varsigma\left(P_{n^{-1 / 2} h_{n}}\right) \doteq n^{-1 / 2} \Sigma \psi\left(\cdot, X_{i}\right)$, which is a variant of (13.13) (i.e., one needs the 'Gateaux derivative' in 'direction' $\hat{F}_{n}$ and in direction $\left.P_{n^{-1 / 2} h_{n}}\right)$, and then (13.19) follows from (13.10). Under these additional assumptions, $\theta\left(\hat{F}_{n}\right)$ is LAM:

(13.20) THEOREM. If $g$ is bounded and uniformly continuous, then

$$
\lim _{c} \lim _{n} \sup _{h \in N_{c}} \int g\left(n^{1 / 2}\left|\theta\left(\hat{F}_{n}\right)-\theta_{n h}\right|\right) d P_{h}^{n}=\int g(T \circ \pi x) d Q_{0} .
$$

(13.21) REMARK. The proof, as usual, proceeds by showing that if $h_{n} \in N_{c}$, then $n^{1 / 2}\left(\theta\left(\hat{F}_{n}\right)-\theta_{n h_{n}}\right)$ converges to $T \circ \pi \circ Z, Z \sim P_{0}$ : i.e., a convergence to the proper limit that is uniform over $N_{c}$. In many applications the neighborhoods $N_{c}$ are very skimpy. In such cases it is important to prove that this convergence is uniform over somewhat broader neighborhoods (not necessarily parametrized only by $H$ ). This will not change the LAM lower bound (cf. (13.17)) and will enhance the desirability of the estimator $\theta\left(\hat{F}_{n}\right)$. The fact that some skimpy neighborhoods cannot be extended much brings up serious questions concerning the ability of the selected minimum distance method to deal with data that suffers from moderately severe departures from the model.

We now discuss a number of examples. We dwell mainly on (13.13) and on the form of $\varsigma, \psi, H, \tau$, so that the general structure becomes apparent; in most cases the identifiability and differentiability hypotheses will be assumed.

EXAMPLE 1. Let $A_{t}$ be the set in $R^{s}$ given by $A_{t}=\left\{u \in R^{s}: u_{i} \leq t_{i}\right\}$ when $t=\left(t_{1}, \ldots, t_{s}\right) \in R^{s}$. Define for any probability $G$ on $R^{s}, \varsigma(G)(t)=G\left\{A_{t}\right\}$. Let $\mu$ 
be a finite measure on $R^{s}$; then $\varsigma(G) \in L^{2}(\mu)$. Fix a parametric family of measures $\left\{P_{\theta}\right\}$, and fix $\theta_{0}$. Then

$$
\left|\zeta(G)-\varsigma\left(P_{\theta}\right)\right|_{\mu}^{2}=\int_{s}\left[G\left(A_{t}\right)-P_{\theta}\left(A_{t}\right)\right]^{2} d \mu,
$$

and so this set up is designed for the situation when 'best fit of model to data' is defined by matching up the 'cumulatives' as closely as possible. Of course, if $s=1$, $\S 6$ treats a special case. To put the present case in the framework of this section, define on $R^{s} \times R^{s}$ the mapping

$$
\psi(t, x)=I_{A_{\mathrm{t}}}(x)-P_{\theta_{0}}\left(A_{t}\right) .
$$

It is easy to see that then

$$
H=\left\{h \in L^{2}\left(d P_{\theta_{0}}\right): \int h d P_{\theta_{0}}=0\right\}=\operatorname{span}\left\{\psi(t, \cdot): t \in R^{s}\right\} .
$$

Then

$$
(\tau h)(t)=\langle\psi(t, \cdot), h\rangle=\int_{A_{t}} h(u) f\left(\theta_{0}, u\right) \nu(d u), \quad h \in H .
$$

So $\tau h$ is actually a continuous function. Moreover, for any $G$,

$$
\varsigma(G)(t)-\varsigma\left(P_{\theta_{0}}\right)(t)=\int \psi(t, x) d\left(G-P_{\theta_{0}}\right)(x),
$$

so (13.13) is an identity for each $n$. If $N_{c}=\{h:|h| \leq c\}$, then for this choice both theorems hold. In fact, more is true: if $G_{n}$ is a sequence of probabilities such that $\int\left|G_{n}\left(A_{t}\right)-P_{\theta_{0}}\left(A_{t}\right)\right|^{2} \mu(d t) \leq c n^{-1 / 2}$, and if $X_{1}, \ldots, X_{n}$ are i.i.d. $G_{n}$, then $n^{1 / 2}\left[\hat{F}_{n}\left(A_{t}\right)-G_{n}\left(A_{t}\right)\right]$ converges in distribution on $L^{2}(\mu)$, implying that $\theta\left(\hat{F}_{n}\right)$ converges uniformly over these neighborhoods to its limit. That is, one can take greatly extended neighborhoods of $P_{\theta_{0}}$, as suggested in the remarks above.

EXAMPLE 2. Here is a variant of Example 1: suppose one wants to fit the model to the data by matching up the characteristic functions as closely as possible (instead of matching up the cumulatives). From a practical point of view one probably wants to estimate a few probabilities, and the method just suggested might not be too helpful for that; but it is conceivable that someone would want to estimate a characteristic function. Assuming this, one may place the problem in the general framework of this section as follows: Define, for any probability $G$ on $R^{s}$, the map $\varsigma(G)(t)=\int \exp i\langle t, x\rangle G(d x)$. Let $\mu$ be a finite measure on $R^{s}$; then $\varsigma(G) \in L^{2}(\mu)$ (complex $L^{2}$ space). Fix a parametric family $\left\{P_{\theta}, \theta \in \Theta\right\}$ and $\theta_{0} \in \Theta$. Then $\left|\zeta(G)-\varsigma\left(P_{\theta}\right)\right|$ is the $L^{2}(\mu)$ distance between the characteristic functions of $G, P_{\theta}$. Define on $R^{s} \times R^{s}$ the map

$$
\psi(t, x)=\exp i\langle t, x\rangle-\int \exp i\langle t, x\rangle P_{\theta_{0}}(d x) .
$$

It is complex valued, and we decline to identify $H$ (it is not hard), the span of $\left\{\psi(t, \cdot): t \in R^{s}\right\}$ in $L^{2}\left(d P_{\theta_{0}}\right)$. The map $\tau$, in the present case, turns out to be

$$
(\tau h)(t)=\int \exp \{i\langle t, x\rangle\} h(x) d P_{\theta_{0}}(x), \quad h \in H .
$$


Again, for any $G$,

$$
\varsigma(G)(t)-\varsigma\left(P_{\theta_{0}}\right)(t)=\int \psi(t, x) d\left(G-P_{\theta_{0}}\right)(x)
$$

so (13.13) is an identity for each $n$. For reasons given in the preceding example, $\theta\left(\hat{F}_{n}\right)$ here is again LAM, the uniform convergence holding over broad neighborhoods like those suggested in that example.

EXAMPLE 3. Suppose the data distribution is $G$ (unknown probability on the line), and we want to fit the model $\left\{P_{\theta}\right\}$ to the data by choosing $\theta$ such that the mean of $P_{\theta}$ is closest to the mean of $G$. This somewhat degenerate situation is discussed briefly here, in preparation for Example 4 (when extended to regression problems, it becomes, essentially, the well-known least squares method). One could use $p$ th-moment instead of mean-the discussion is essentially the same.

To put the foregoing into our framework, define $\varsigma(G)=\int x G(d x)$. Note that $\varsigma$ is not defined for very many $G$. In this case $L^{2}(Y, \mu)$ will be the real line with the usual distance. As usual, fix $\theta_{0}$. Define $\psi(t, x)$ on $R^{\prime} \times R^{\prime}$ by $\psi(t, x)=x-$ $c$ for all $t$, where $c=\int x d P_{\theta_{0}}(x)$. Then $H$ is the one-dimensional subspace of $L^{2}\left(d P_{\theta_{0}}\right): H=\left\{\lambda(x-c): \lambda \in R^{\prime}\right\}$. The mapping $\tau$ is $\tau\{\lambda(\cdot-c)\}=\lambda \sigma^{2}$, where $\sigma^{2}=\int(x-c)^{2} P_{\theta_{0}}(d x)$; here $\lambda(\cdot-c)$ is a generic element of $H$. The measure $P_{0}$ is $N\left(0, \sigma^{2}\right)$. The differentiability assumption becomes the ordinary differentiability at $\theta_{0}$ of $\theta \rightarrow \int x d P_{\theta}(x)$; nonsingularity just means that the value of the derivative at $\theta_{0}$ is not zero. Identifiability means essentially that different $P_{\theta}$ 's must have different means. Again, in this example, (13.13) is trivial, holding without the need of taking any limit.

It is important to notice that here our usual neighborhoods $N_{c}=\{h \in H:|h| \leq$ c) are one dimensional. Assuming $\int(x-c)^{2} P_{\theta_{0}}(d x)<\infty$, the normalized sample mean converges, under $P_{h_{n}}^{n}, h_{n} \in N_{c}$, to $P_{0}$. Since in the present case $\pi$ is the identity, the minimum distance estimate of $\theta$ is $\bar{X}$, the sample mean. The development so far is unsatisfactory, since the choice of $N_{c}$ dictated by (13.15)ff is only one dimensional, so the allowable data distributions are extremely limited (and most likely do not include any $P_{\theta}$ 's other than $P_{\theta_{0}}$ !). Therefore, to get a useful result, one must attempt to show that $\bar{X}$ converges over rather broad neighborhoods of $P_{\theta_{0}}$. If one attempts to use the $N_{c}$ of, say, Example 1 then such convergence will fail: typically, measures of the form $f_{\theta_{0}}\left(1+h n^{-1 / 2}\right)$, where $h$ satisfies only $\int f_{\theta} h=0$, will not even have a mean; so the "neighborhoods" of Example 1 are too big. An intermediate neighborhood $N_{c}$ (that works!) is of the form $\left\{h: \int h f_{\theta}=0\right.$, support $h \subset\{-c, c],|h| \leq c\}$. This will not be completely satisfactory, since it will still probably not contain $P_{\theta}$ for $\theta$ close to $\theta_{0}$. Another possibility is $\left\{h: \int h f_{\theta}=0\right.$, $\left.|h|^{2} \leq c, \int x^{2} h^{2} f_{\theta_{0}} \leq c\right\}$.

EXAMPLE 4. This example presents a very reasonable minimum distance method for the situation where the data distribution is believed to be not too different from those posited by the model $\left\{P_{\theta}\right\}$. Suppose the measures are on the line. The idea is to find $\theta$ so that the distance between the cdf's and first $k$ moments of $P_{\theta}, G$ is as small as possible: that is, find $\theta$ that achieves the inf:

$$
\inf _{\theta}\left\{\left|G-P_{\theta}\right|^{2}+\sum_{i=1}^{k}\left[\int x^{i} d G-\int x^{i} d P_{\theta}\right]^{2}\right\}
$$


where $\left|G-P_{\theta}\right|$ is an $L^{2}$-norm between the cdf of $G, P_{\theta}$. We show next how to formulate this using the structure developed earlier in this section. Let $e_{0}, e_{1}, \ldots, e_{k}$ be an orthonormal set in $L^{2}([0,1], d x)$ (unit interval with Lebesgue measure). Let $G(t), P_{\theta}(t)$ denote cdf's of $G, P_{\theta}$. Fix $\theta_{0}$. Let $m$ be a finite measure on the line, so $G(t) \in L^{2}(m)$. Define $Y=[0,1] \times R^{1}, d \mu=d x \times d m$. The $L^{2}(Y ; d \mu)$-valued elements $\varsigma$ will now be defined by

$$
\varsigma(G)\left(t_{1}, t_{2}\right)=e_{0}\left(t_{1}\right) G\left(t_{2}\right)+\sum_{i=1}^{k} e_{i} \int x^{i} d G(x) .
$$

Then

$$
\left|\varsigma(G)-\varsigma\left(P_{\theta}\right)\right|_{\mu}^{2}=\int\left[G(t)-P_{\theta}(t)\right]^{2} m(d t)+\sum\left[\int x^{i} d\left(G-P_{\theta}\right)\right]^{2},
$$

so the $\theta$ that achieves $\inf _{\theta}\left|\varsigma(G)-\varsigma\left(P_{\theta}\right)\right|$ is the one we want. Define, for $t=\left(t_{1}, t_{2}\right) \in$ $Y$,

$$
\psi(t, x)=e\left(t_{1}\right)\left[I_{t_{2}}(x)-P_{\theta_{0}}\left(t_{2}\right)\right]+\sum_{1}^{k} e_{i}\left(t_{1}\right)\left(x^{i}-c_{i}\right),
$$

where $c_{i}=\int x^{i} P_{\theta_{0}}(d x)$. It is clear that $H=\left\{h: \int h^{2} d P_{\theta_{0}}<\infty, \int h d P_{\theta_{0}}=0\right\}$, and it is fairly routine to find $\tau, P_{0}$, etc., for this particular example; as in all other cases so far (13.13) is trivial. For reasons given in Example 3, one cannot use $N_{c}=\{h \in H:|h| \leq c\}$ here: $\varsigma\left(P_{h n^{-1 / 2}}\right)$ is not defined for most of these $h$. One workable possibility is $N_{c}=\{h \in H:|h| \leq c$, support $h \subset[-c, c]\}$. Then $N_{c}$ increases with $c, N_{c}$ is convex, and $\bigcup N_{c}$ is dense in $H$. With the foregoing choice (assuming as usual the differentiability and identifiability hypotheses), $\theta\left(\hat{F}_{n}\right)$ is LAM.

EXAMPLE 5. In this example let us try to fit the model to the data by matching up quantiles. This was done in detail in $\S 9$; here, as preparation for Example 6, we indicate only how the result of $\S 9$ fits into the present framework. We do not dwell on regularity hypotheses; for these, see $\S 9$.

Define, for a distribution $G$ on the line, $\varsigma(G)(t)=G^{-1}(t) I_{[\alpha, \beta]}(t)$, where $t \in$ $[0,1], 0<\alpha<\beta<1$. Let $Y=[\alpha, \beta]$ and let $\mu$ be a finite measure on $Y$; then $\varsigma(G) \in L^{2}(Y, d \mu)$ (assuming support $G$ is sufficiently large see $\S 9$ ). Bring in the parametric family $\left\{P_{\theta}\right\}$ of probabilities on the line and fix $\theta_{0}$. Define on $[\alpha, \beta] \times R$ the map

$$
\psi(t, x)=c_{t}\left[I_{A_{t}}-t\right]
$$

where

$$
c_{t}=1 / f\left(F^{-1}(t)\right), \quad A_{t}=\left(-\infty, F^{-1}(t)\right], \quad F=\text { cdf of } P_{\theta_{0}} .
$$

It is clear that

$$
H=\operatorname{span}\{\psi(t, \cdot), t \in[\alpha, \beta]\}=\left\{h: \int h^{2} f\left(\theta_{0}\right)<\infty, \int h f_{\theta_{0}}=0\right\} .
$$

The map $\tau$ is given by

$$
(\tau h)(t)=c_{t} \int^{F^{-1}(t)} h(u) f_{\theta_{0}}(u) d u
$$


The covariance kernel

$$
K(s, t)=\langle\psi(t, \cdot), \psi(s, \cdot)\rangle=c_{t} c_{s}[s \wedge t-s t]
$$

on $[\alpha, \beta] \times[\alpha, \beta]$ and this identifies $P_{0}$ as the distribution of $c_{t} W^{0}(t), \alpha \leq t \leq \beta$, where $W^{0}$ is the usual Brownian bridge on $[0,1]$. Finally, note that if $h \in H$ is sufficiently smooth,

$$
\lim _{n} n^{1 / 2}\left[\varsigma\left(P_{h^{-1 / 2} h}\right)-\varsigma\left(P_{\theta_{0}}\right)\right]=\tau h
$$

see $\S$. This is the first example where (13.13) is not an identity. As pointed out in $\S 9$, the neighborhood system $N_{c}$ has to be chosen with some care to ensure (13.13) for $h \in N_{c}$. That $\theta\left(\hat{F}_{n}\right)$ is here optimal was discussed in $\S 9$.

EXAMPLE 6. In this example we merely point out a number of interesting possibilities based on examples above.

(a) Instead of basing the notion of 'best fit' on the quantile function as in Example 6 , one could pick a particular quantile (e.g., the median) and define $\theta$ to be estimated as the one that makes (say) median $P_{\theta}$ closest to median $G$, where $G$ is the unknown data distribution. Evidently this is the analogue for quantile functions of Example 3. More generally, one may proceed as follows. Let $Y$ be some space, and for each $t \in Y$, let $a(t, d x)$ be a measure on the line. The proposal then is to base a minimum distance method on the elements $\varsigma(G)(t)=\int G^{-1}(u) a(t ; d u)$, where $G$ is a cdf on the line. If $a$ is independent of $t$, then $\zeta$ is the usual $L$ functional, common in robustness studies $(a(t, d x)=$ unit mass at $1 / 2$ gives median, for example). Many interesting possibilities arise on letting $a(t, d x)$ vary with $t(a(t, d x)=$ unit mass at $\{t\}$ gives Example 6). The methods of this section are easily adapted to analyze such functionals.

(b) Picking up one of the suggestions of Example 6(a), we can return to Examples 1-3 and introduce kernels $k(t, x)$, where $t$ is in some appropriate space, and define for a probability distribution $G, \varsigma(G)(t)=\int k(t, x) G(d x)$. The choices $k(t, x)=I_{A_{t}}(x), A_{t}=\{u: u \leq t\}, k(t, x)=\exp \{i\langle t, x\rangle\}$, and $k(t, x)=x^{P}$ yield, respectively, Examples 1-3. More fun is possible on letting $t$ vary in appropriate infinite-dimensional Hilbert spaces. Of course, $\varsigma(G)(\cdot)$ should belong to some $L^{2}(\mu)$-space. As another variant, one may define $\zeta$ to be a favorite $M$-functional defined on distributions $G$ (not necessarily distributions on the line). The minimum distance methods based on these choices of $\varsigma$ are easily analyzed by the theory of this section.

(c) Finally, one may desire to fit the model to the data by matching up both the cdf's and the quantiles. Here is one possibility. Let $t=\left(t_{1}, t_{2}, t_{3}\right)$ denote a point in the space $Y=R^{\prime} \times[\alpha, \beta] \times[0,1]$, where $0<\alpha<\beta<1$. Let $m_{1}$ be a measure on $R^{\prime}, m_{2}$ a measure on $[\alpha, \beta]$ and set $\mu=m_{1} \times m_{2} \times m_{3}, m_{3}=$ Lebesgue measure on $[0,1]$. Define, for any probability $F$,

$$
\varsigma(F)(t)=F\left(t_{1}\right) e_{1}\left(t_{3}\right)+F^{-1}\left(t_{2}\right) e_{2}\left(t_{3}\right),
$$

where $e_{1}, e_{2}$ are orthonormal in $L^{2}([0,1])$. Then if ||$_{\mu}$ denotes $L^{2}(\mu)$-norm,

$$
|\varsigma(F)|_{\mu}^{2}=\int F^{2}\left(t_{1}\right) d m_{1}\left(t_{1}\right)+\int\left[F^{-1}\left(t_{2}\right)\right]^{2} m_{2}\left(d t_{2}\right) .
$$

The theory of this section then easily applies to the minimum distance procedure based on $\varsigma(F) \in L^{2}(\mu)$. 
EXAMPLE 7. Up to now we have assumed $Y$ is a Euclidean space. The theory can be pressed further; for illustration, let us take $Y$ to be $L^{2}\left(R^{\prime}, d m\right)$. A modest amount of care is needed to carry through the forthcoming suggestion rigorously, but let us proceed heuristically. Let us define a functional $\varsigma$ on (certain) probabilities $G$ on the line by $\varsigma(G)(t)=\int t(u) G(d u)$ if $t \in Y$. This will, of course, not be defined for all $G$-it depends on what $m$ is. Let $\left\{e_{i}\right\}$ be an orthonormal set in $Y$ and let $\mu$ be the Gaussian measure on $Y$ which is the distribution of $\Sigma \lambda_{i} X_{i} e_{i}$, where $\Sigma \lambda_{i}^{2}<\infty$ and the $X_{i}$ are i.i.d. $N(0,1)$. Readers familiar with the theory of Gaussian measures on Hilbert spaces can give more elegant descriptions of $\mu$. In the formalism of this section, the proposed $\psi(t, x)=t(x)$; there are problems here with joint measurability, but never mind. If $\left\{P_{\theta}\right\}$ is our usual parametric family, then the minimum distance problem is to estimate $\theta(G)$, the point which achieves the infimum in

$$
\inf _{\theta}\left|\zeta(G)-\varsigma\left(P_{\theta}\right)\right|_{\mu}^{2}=\inf _{\theta} \sum \lambda_{1}^{2}\left[\int e_{i} d\left(P_{\theta}-G\right)\right]^{2} .
$$

It is probably easier to analyze this metric directly, rather than representing it in $\psi$-form; nevertheless, we can easily believe now that asymptotic normality and LAM results do indeed hold in the present case. The details are not difficult, given the structural results of $\S \S 3,5$.

XIV. Estimating a mixture. Except for technicalities, the example of this section is conceptionally much simpler than that of $\S 6$. It is included to illustrate the usefulness of a parameter set $\boldsymbol{\theta}$ which is an infinite-dimensional Hilbert space.

To describe the problem let $F(t, u)$ be a measurable function of $R^{1} \times R^{d}$ such that for each $u, t \rightarrow F(t, u)$ is a cdf of the line. The function $F$ is assumed known. If $\nu$ is a probability on $R^{d}$, define the "mixture" $F(t, \nu)$ by

$$
F(t, \nu)=\int F(t, u) \nu(d u)
$$

so $F(\cdot, \nu)$ is again a cdf on $R^{1}$. Let $X_{1}, \ldots, X_{n}$ be i.i.d. with common distribution given by $F(\cdot, \nu)$ for some $\nu$. The statistical problem is to estimate the mixing distribution $\nu$. This section gives a LAM solution to a subset of this problem using minimum distance methods. Since the technicalities are lengthy, we illustrate only the general approach.

To describe the approach first bring in a known sigma-finite measure $\mu$ on $R^{d}$. For a given mixing distribution $\nu$, let $\theta$ be its cdf on $R^{d}$ :

$$
\theta(a)=\nu\{u: u \leq a\} .
$$

Identify $\nu$ with $\theta$ and assume that the exact set of available mixed cdf's is given by the recipe

$$
F(t, \theta)=\int(\theta)(a) \psi(t, a) \mu(d a)+g(t)
$$

where $\psi$ is a known jointly measurable function on $R^{1} \times R^{d}, g$ is a known function on $R^{1}$, and $\theta$ is some point in $L^{2}\left(R^{d}, \mu\right)$. In many examples, (14.3) is obtained from (14.1) by an integration by parts, and $\mu$ becomes either Lebesgue measure or a counting measure; nevertheless, (14.3) typically restricts both $F(t, u)$ and the 
available mixing measures. For convenience, we assume $g=0$. Also assume that $T: \theta \rightarrow F(\cdot, \theta)$ is a continuous linear operator into $L^{2}(m)$, where $m$ is some sigmafinite measure on $R^{1}$. With these assumptions, the proposed estimate $\hat{\theta}_{n}$ is the minimum distance estimator determined by

$$
\inf _{\theta \in L^{2}(\mu)}\left|\hat{F}_{n}-T(\theta)\right|_{m}=\left|\hat{F}_{n}-T\left(\hat{\theta}_{n}\right)\right|_{m},
$$

where ||$_{m}$ is the norm of $L^{2}(m)$, and $\hat{F}_{n}$ is the empirical cdf. This estimate has been studied by Burman (1983), who used methods from integral equations; we develop instead our slightly more general approach using the structural theorems of $\S \S 3,5$.

Because of (2.13) and the linearity of $T$, it is immediate that

$$
\hat{\theta}_{n}=K^{-1} T^{*} \hat{F}_{n},
$$

provided we assume $K^{-1}$ exists. In the present situation, (14.6)

$$
\begin{aligned}
(T \theta)(t) & =\int \theta(u) \psi(t, u) \mu(d u), \\
\left(T^{*} V\right)(a) & =\int V(t) \psi(t, a) m(d t), \\
(K \theta)(s) & =\int \theta(a) U(a, s) \mu(d a), \quad \text { where } U(a, s)=\int \psi(t, a) \psi(t, s) m(d t) .
\end{aligned}
$$

If $\theta_{0}$ is fixed and $X_{1}, \ldots, X_{n}$ are i.i.d. $T \theta_{0}$, then (14.5) implies that

$$
n^{1 / 2}\left(\hat{\theta}_{n}-\theta_{0}\right) \Rightarrow Y
$$

in distribution on $L^{2}(\mu)$. Here $Y$ is a Gaussian random element of $L^{2}(\mu)$ with covariance operator specified by $(2.15)$, with $S$ there given by the covariance operator of $n^{1 / 2}\left(\hat{F}_{n}-F\left(\cdot, \theta_{0}\right)\right)$. Thus the minimum distance estimate is asymptotically normal.

To complete this section we sketch briefly the LAM framework. Fix cdf $\theta_{0}$ having density $f: \theta_{0}(t)=\int^{t} f$. Let $H=\left\{h: \int f h=0\right\}, \tau_{0} h(t)=\int^{t} h f, \tau h(t)=$ $\int \psi(t, u)\left(\tau_{0} h\right)(u) \mu(d u), \tau: h \rightarrow L^{2}(m)$. Simple considerations show that $(\tau, H, \overline{\tau H})$ is an abstract Wiener space, $P_{0}$ being the distribution of the process $T Y$ of (14.7). If the local perturbations of the distributions of the $X_{i}$ at time $n$ are

$$
\int \psi(t, u)\left(\theta_{0}+n^{-1 / 2} \tau_{0}\right)(u) \mu(d u),
$$

then one obtains the LAM property as in $\S 6$.

XV. Proof of (2.8), (3.6).

PROOF OF (2.8). The first point in the proof is that

$$
\inf _{|\theta| \leq c}\left|\xi_{n}(\theta)\right|=\inf _{\theta}\left|\xi_{n}(\theta)\right|
$$

for all $n$, with probability approaching 1 as $c \rightarrow \infty$. To see this, note that since $\left|\xi_{n}(\theta)\right| \geq\left|\xi_{n}(\theta)-\xi_{n}\left(\theta_{0}\right)\right|-\left|\xi_{n}\left(\theta_{0}\right)\right|$, it follows that

$$
\inf _{|\theta|>c}\left|\xi_{n}(\theta)\right| \geq \inf _{|\theta|>c}\left|\xi_{n}(\theta)-\xi_{n}\left(\theta_{0}\right)\right|-\left|\xi_{n}\left(\theta_{0}\right)\right| \text {. }
$$


Because of (2.1), (2.4), the right side of this expression goes to $+\infty$ in probability as $c \uparrow \infty$, proving (14.1).

Next, fix $c$ (large) and let $\hat{\theta}_{n}$ satisfy

$$
\inf _{|\theta| \leq c}\left|\xi_{n}(\theta)\right|=\left|\xi_{n}\left(\hat{\theta}_{n}\right)\right|
$$

Since $\left|\hat{\theta}_{n}-\theta_{0}\right|<2 c$,

$$
\xi_{n}\left(\hat{\theta}_{n}\right)=\xi_{n}\left(\theta_{0}\right)+\delta_{n}\left\langle\hat{\theta}_{n}-\theta_{0}, \eta\right\rangle+o(1)
$$

by (2.2). It is easy to see that, for all large $c$,

$$
\inf _{\left|\theta-\theta_{0}\right|<c}\left|\xi_{n}\left(\theta_{0}\right)+\delta_{n}\left\langle\theta-\theta_{n}, \eta\right\rangle\right|=\inf _{\theta}\left|\xi_{n}\left(\theta_{0}\right)+\delta_{n}\left\langle\theta-\theta_{0}, \eta\right\rangle\right|,
$$

so any point $\tilde{\theta}_{n}$ that achieves the inf in (14.4) satisfies

$$
\begin{gathered}
\delta_{n}\left\langle\tilde{\theta}_{n}-\theta_{0}, \eta\right\rangle=-\pi \xi_{n}\left(\theta_{0}\right), \quad\left|\tilde{\theta}-\theta_{0}\right| \leq c, \\
\xi_{n}\left(\tilde{\theta}_{n}\right)=(1-\pi) \xi_{n}\left(\theta_{0}\right)+o(1) .
\end{gathered}
$$

By (14.1) and differentiability,

$$
\left|\xi_{n}\left(\hat{\theta}_{n}\right)\right|=\left|\xi_{n}\left(\tilde{\theta}_{n}\right)\right|+o_{n}(1) .
$$

By the Pythagorean theorem,

$$
\left|\left\langle\hat{\theta}_{n}-\theta_{0}, \eta\right\rangle-\left\langle\tilde{\theta}_{n}-\theta_{0}, \eta\right\rangle\right|^{2}=\left|\xi_{n}\left(\hat{\theta}_{n}\right)\right|^{2}-\left|\xi_{n}\left(\tilde{\theta}_{n}\right)\right|^{2}+o(1)=o(1) .
$$

This implies

$$
\xi_{n}\left(\hat{\theta}_{n}\right)=\xi_{n}\left(\tilde{\theta}_{n}\right)=(1-\pi) \xi_{n}\left(\theta_{0}\right)+o(1)
$$

and

$$
\delta_{n}\left\langle\hat{\theta}_{n}-\theta_{0}, \eta\right\rangle=-\pi \xi_{n}\left(\theta_{0}\right)+o(1),
$$

which is the desired result.

PROOF OF (3.6). As remarked in $\S 3$, one proves (3.6) by applying (2.8) to the processes $\xi_{n}^{0}(\theta)=n^{1 / 2}\left[\xi_{n}\left(\theta_{0}+\theta n^{-1 / 2}\right)\right]$. One need only check the differentiability, boundedness, and identifiability hypotheses of $\S 2$ when applied to $\xi_{n}^{0}(\theta)$. We indicate here only identifiability, since the other two are clear. For this take $\theta_{0}=0$ for simplicity and suppose that $\inf _{n} \inf _{|\theta|>c}\left|\xi_{n}\left(\theta_{n-1 / 2}\right)\right| n^{1 / 2}$ remains bounded as $c \uparrow \infty$; we deduce a contradiction. Let $c$ increase to $+\infty$ through the integers $k$; let $n_{k}, \theta_{k},\left|\theta_{k}\right| \geq k$, achieve the inf when $c=k$, so that $\xi_{k}\left(\theta_{k} n_{k}^{-1 / 2}\right) n_{k}^{1 / 2}$ is bounded as $k \rightarrow \infty$. The identifiability assumption of $\S 3$ then implies that $\theta_{k} / n_{k}^{1 / 2} \rightarrow 0$ since $\xi_{k}\left(\theta_{k} n_{k}^{-1 / 2}\right) \rightarrow 0$. Therefore, since $\left|\theta_{k}\right| \geq k, n_{k} \rightarrow+\infty$. Moreover, since $\theta_{k} n_{k}^{-1 / 2}$ goes to zero, the differentiability assumption of $\S 3$ applies, so

$$
\xi_{k}\left(\theta_{k} n_{k}^{-1 / 2}\right)=\xi_{k}(0)+\left\langle\theta_{k}, \eta\right\rangle n_{k}^{-1 / 2}+o\left(\theta_{k} n_{k}^{-1 / 2}\right) .
$$

Therefore, as $k \rightarrow \infty$ with probability approaching 1 ,

$$
\left|\xi_{n}\left(\theta_{k} n_{k}^{-1 / 2}\right)\right| \geq \frac{1}{2} c_{0}\left|\theta_{k} n_{k}^{-1 / 2}\right|-\left|\xi_{n}(0)\right|,
$$

using nonsingularity $\left(|\langle\theta, \eta\rangle| \geq c_{0}|\theta|\right)$. The assumed boundedness of $n_{k}^{1 / 2} \xi_{n}\left(\theta_{k} n_{k}^{-1 / 2}\right)$ and (15.9) imply that $\left\{\theta_{k}\right\}$ is bounded, a contradiction. 


\section{REFERENCES}

1. R. J. Beran, Efficient robust estimation for parametric models, Z. Wahrsch. Verw. 55 (1981), 91-108.

2. D. Blackwell, Equivalent comparisons of experiments, Ann. Math. Statist. 24 (1953), 265-272.

3. E. Bolthausen, Convergence in distribution of minimum distance estimators, Metrika 24 (1977), 215-227.

4. Prabir Burman, Estimation of the mixing distribution, 1983 (preprint).

5. R. G. Davies, Asymptotic inference in time series, Adv. in Appl. Probab. 5 (1973), 469-497.

6. J. L. Doob, Stochastic processes, Wiley, 1953.

7. J. Hajek, Local arymptotic minimax and admissibility in estimation, Proc. Sixth Berkeley Sympos., Vol. 1, 1972, pp. 170-194.

8. P. J. Huber, Robust statistics, Wiley, New York, 1981.

9. A. Ibragimov, Estimation of the spectral function of a stationary Gaussian process, Theory Probab. Appl. 8 (1963), 391-430.

10. H. L. Koul and T. de Wet, Minimum distance estimation in a linear regression model, Ann. Statist. 11 (1983), 921-932.

11. V. N. La Riccia, Asymptotic properties of weighted $L^{2}$ quantile distance estimators, Ann. Statist. 10 (1982), 621-624.

12. L. Le Cam, Sufficiency and arymptotic rufficiency, Ann. Math. Statist. 35 (1964), 1419-1455.

13. _ Théorie asymptotique de la décision statistique, Presses Univ. Montréal, Montréal, 1969.

14. __ Limits of experiments, Proc. Sixth Berkeley Sympos., Vol. 1, Univ. of California Press, Berkeley, 1972.

15. __, Convergence of estimates under dimensionality restrictions, Ann. Statist. 1 (1973), 38-53.

16. P. W. Millar, Asymptotic minimax theorems for the sample distribution function, Z. Wahrsch. Verw. Gebiete 48 (1979), 233-252.

17. __ Robust estimation via minimum distance methods, Z. Wahrsch. Verw. Gebiete 55 (1981), 73-89.

18. W. C. Parr, Minimum distance estimation: a bibliography, Comm. Statist. A-Theory Methods 10 (1981), 1205-1224.

19. W. C. Parr and W. R. Schucany, Minimum distance and robust estimation, J. Amer. Statist. Assoc. 75 (1980), 616-624.

20. W. C. Parr and T. de Wet, On minimum weighted Cramer-von Mises statistic estimation, 1980 (preprint).

21. K. R. Parthasarathy, Probability mearures on metric spaces, Academic Press, 1967.

22. D. Pollard, The minimum distance method of testing, Metrika 27 (1980), 43-70.

23. G. G. Roussas, Contiguous probability mearures: some applications in statistics, Cambridge Univ. Press, 1972.

24. R. J. Serfling, Approximation theorems of mathematical statistics, Wiley, 1980.

25. G. Shorack, Convergence of quantile and spacings processes with applications, Ann. Math. Statist. 43 (1972), 1400-1411.

26. J. Wolfowitz, The minimum distance method, Ann. Math. Statist. 28 (1957), 75-88.

Department of Statistics, University of CAlifornia, Berkeley, CAlifornia 94720 